“ C 2019 IEEE. Personal use of this material is permitted. Permission from IEEE must be obtained for all other uses, in any current or future media, including

reprinting/republishing this material for advertising or promotional purposes, creating new collective works, for resale or redistribution to servers or lists, or reuse of any copyrighted component of this work in other works." 


\title{
Low-Complexity Multiuser Receiver for Massive Hybrid Array mmWave Communications
}

\author{
Hang $\mathrm{Li}^{(}{ }^{(}$, Thomas Q. Wang $\left.{ }^{(}\right)$, Xiaojing Huang $\left.{ }^{(}\right)$, Senior Member, IEEE, \\ J. Andrew Zhang ${ }^{\circledR}$, Senior Member, IEEE, and Y. Jay Guo ${ }^{\circledR}$, Fellow, IEEE
}

\begin{abstract}
In this paper, we study the low complexity reception of multiuser signals in uplink millimeter wave (mmWave) communications using a partially connected hybrid antenna array. Exploiting the mmWave channel property, we propose a lowcomplexity user-directed multiuser receiver with three novel schemes for allocating subarrays to users. This receiver only requires the knowledge of angles-of-arrival (AoAs) for dominating paths and a small amount of equivalent channel information instead of perfect channel state information. For comparison, we also derive a successive interference cancellation-based solution as a performance benchmark. We design two types of reference signals with the channel estimation method to enable efficient and simple estimation for AoA and equivalent baseband channel. Also, we provide analytical results for the performance of the AoA estimation, using the lower bounds of mean square errors in line-of-sight dominated mmWave channels. The simulation results validate that the proposed channel estimation method is effective when employed in combination with a zero-forcing equalizer.
\end{abstract}

Index Terms-Hybrid antenna array, subarray, angle-ofarrival estimation, line-of-sight, and mmWave communications.

\section{INTRODUCTION}

W ITH balanced system performance, implementation complexity and hardware cost, millimeter wave (mmWave) hybrid antenna array [1]-[4] is regarded as an enabling technology for $5 \mathrm{G}$ wireless communications. A hybrid array can be categorized into the fully-connected structure [5] where each antenna connects to multiple phase shifters and all radio-frontend (RF) chains, and the partiallyconnected structure [2] where each antenna only connects to one phase shifter and one RF chain. The former employs full beamforming (BF) gain for each RF chain such that better performance can be achieved. However, it requires too many phase shifters particularly for massive array, thus greatly increasing the hardware implementation complexity and making the integration into a chip difficult. On the other

Manuscript received July 31, 2018; revised November 30, 2018; accepted January 13, 2019. Date of current version May 15, 2019. This work was supported by Australian Research Council Discovery Project DP160101693. The associate editor coordinating the review of this paper and approving it for publication was E. Basar. (Corresponding author: Hang Li.)

The authors are with the Global Big Data Technologies Centre, University of Technology Sydney, Ultimo, NSW 2007, Australia (e-mail: hang.li@uts.edu.au; qian.wang@uts.edu.au; xiaojing.huang@uts.edu.au; andrew.zhang@uts.edu.au; jay.guo@uts.edu.au). hand, the latter uses much less phase shifters and is more suitable for practical hardware implementation at the cost of small performance loss.

Hybrid precoding and/or combining for mmWave hybrid array include both analog and digital BF, which are designed either independently or jointly for optimizing the system performance [6]. The sum-rate of a multiuser multiple-input multiple-output (MIMO) communication system with a hybrid array is determined by the equivalent baseband channel [7], which includes actual physical channels and analog BF. As a result, analog $\mathrm{BF}$ has more significant impact on the sum-rate of systems. Since analog BF adopts analog phase shifters with constant amplitude, the sum-rate optimization problem is very challenging and usually results in high complexity. Most existing optimization methods require the channel state information (CSI) at each antenna element, which is hard to obtain in practice. This is because 1) the analog BF prevents the channel estimation from directly accessing each antenna output, 2) the massive antennas lead to high-dimensional channel parameters to be estimated, and 3) the signal-to-noise ratio (SNR) for channel estimation is very low due to the large propagation loss.

Most of prior works on analog $\mathrm{BF}$ design and channel estimation in mmWave multiuser MIMO systems consider the fully-connected array [5], [7]-[12]. Assuming perfect CSI, Sohrabi and $\mathrm{Yu}$ [5] seek to find the optimal analog BF by decoupling and iterating the designs of analog $\mathrm{BF}$ and digital precoder. To reduce the complexity incurred by iterations, the works in [9] and [10] studied a low-complexity phase-only eigen-BF scheme, which exploits maximum ratio combining and matched filtering for analog BF. Combined with block diagonalization based digital precoding, the phase-only based analog BF was also investigated in [7] with equal gain transmission. To alleviate the needs for excessive CSI, [11] proposed a low-complexity two-stage multiuser hybrid precoding algorithm, which efficiently reduces the training and feedback overhead while achieving performance similar to that of digital unconstrained precoding. In the first stage, the analog BF design is based on maximizing the desired user's signal power by exhaustive beam searching using the designed codebook, while ignoring the interference among users. In the second stage, the digital zero-forcing $(\mathrm{ZF})$ precoding is designed through explicit effective CSI feedback. Limited feedback and CSI quantization resolution may cause system performance degradation. In order to avoid the feedback, [12] designed the 
analog BF by exploiting multiple-frequency tones to estimate the strongest angles-of-arrival (AoAs), which is applicable to both non-sparse and sparse mmWave channels. However, its performance is subject to the searching accuracy and potentially increases the filter design complexity. In [13], an adaptive compressed sensing (CS) based solution was proposed to iteratively estimate the mmWave channel parameters by exploiting the sparse nature of mmWave channel. It was formulated as a sparse recovery problem where the measurement matrix is constructed with the training precoding/combining matrices. The received signal is a linear function of sparse channel vector including path gains of the quantized spatial frequencies. The non-zero elements in the channel vector are used to estimate the AoAs. The training beamforming vectors constructed by using hierarchical multi-resolution codebook are used to generate the beams with different directions and beamwidths. A similar algorithm was applied to mmWave massive MIMO systems with lens antenna array [14]. However, such CS based algorithms require repeatedly selecting the codewords for the transmitter to narrow down the beam. This will introduce a long delay, and the estimation accuracy depends on the resolutions of the codebooks. In addition, it may not be applicable to non-sparse mmWave channels [12].

There have been only limited works for the partiallyconnected array. Dai et al. [15] and Gao et al. [16] proposed a successive interference cancellation (SIC) approach to iteratively optimize the analog $\mathrm{BF}$ and achievable rate of each subarray, which is proved to be a near-optimal solution for single user MIMO with multiple data streams. Our previous works [17] studied some low-complexity user-directed analog $\mathrm{BF}$ schemes, where the channel correlation between different users can be efficiently reduced by allocating different subarrays to different users. Although the user-directed schemes achieve excellent performance, the CSI at each antenna element is still required to be known. In [18], a low-complexity two-stage multiuser hybrid precoding scheme with dynamic beam assignment was proposed for the partially-connected array, which achieves high system throughput through effective channel information feedback. However, it uses hierarchical beam search, resulting in high search overhead. In [19], two low-complexity uplink multiuser reception schemes were proposed, which only require users' AOAs, but no efficient AoA estimation scheme is provided.

In this paper, we study a low-complexity multiuser receiver for massive hybrid array mmWave uplink communications, where we focus on the interleaved configuration for the proposed schemes. In the interleaved configuration, the partiallyconnected hybrid arrays consist of multiple interleaved subarrays [2] (refer to Fig. 2). Our schemes only require users' AOAs from the line-of-sight (LOS) paths and a small amount of equivalent channel information. The proposed receiver simplifies analog BF implementation, saving the cost for obtaining CSI significantly. Our main contributions are summarized as follows.

- We propose a low-complexity user-directed multiuser receiver which can apply one of three optimized schemes to allocate subarrays to users. We also derive an SIC based solution as its performance benchmark. Simulation results demonstrate that the achievable sum-rates of the proposed schemes are close to that of SIC when LOS is dominant.

- Inspired by the differential beam tracking (DBT) AoA estimation algorithm [20], we propose a long reference signal design for multiuser AoA estimation, which effectively removes multiuser interference. We also derive the lower bounds for the recursive mean square error (MSE) of AoA estimation.

- Based on the optimized allocation of subarrays to users, we carry out the least square (LS) equivalent channel estimation with specifically proposed short reference signals. The estimation performance is evaluated by its MSEs. Simulation results show that our schemes with the proposed channel estimation method provide a good balance between the performance and implementation complexity for mmWave MIMO systems with massive hybrid array.

The remainder of this paper is organized as follows. Section II describes mmWave multiuser MIMO systems. Section III proposes three novel user-directed BF schemes for a partially-connected hybrid array. Section IV details the proposed channel estimation method, sketches the DBT algorithm for single user AoA estimation, and proposes a long reference signal design for multiuser AoA estimation. Section $\mathrm{V}$ derives the recursive MSE lower bounds for AoA estimation in LOS-dominated mmWave channels for performance evaluation. In Section VI, simulation results demonstrating the performance of the studied schemes are presented, before concluding the paper in Section VII.

The following notations are used throughout this paper. $\mathbf{A}$, a and $a$ stand for a matrix, a column vector and a scalar, respectively; $\mathbf{A}^{T}, \mathbf{A}^{H}$ and $\|\mathbf{A}\|_{F}$ denote the transpose, conjugate transpose and Frobenius norm of $\mathbf{A}$, respectively; $\mathbf{I}_{N}$ is the identity matrix with $N$ dimensions; $\mathcal{C N}(\mathbf{m}, \mathbf{V})$ represents a complex Gaussian random vector with mean $\mathbf{m}$ and covariance matrix $\mathbf{V}$. Further, the notations $\log (\cdot), \mathbb{E}[\cdot]$, $\operatorname{Tr}(\cdot)$ and $|(\cdot)|$ represent the logarithmic, expectation, trace and determinant or absolute value depending on context of $(\cdot)$, respectively; $\arg \{\cdot\}$ and $\operatorname{sign}\{\cdot\}$ denote the argument and sign of $(\cdot)$, respectively; $\mathbb{C}^{M \times N}$ denotes the space of all $M \times N$ matrices with complex entries.

\section{SySTEM MODEL}

\section{A. Signal Model}

Consider a multiuser mmWave uplink transmission system that consists of one base station (BS) receiver with a partially-connected hybrid array [2] and $J$ users. As shown in Fig. 1, the receiver is composed of the blocks of analog $\mathrm{BF}, \mathrm{RF}$ chains and baseband signal processing. Analog BF is implemented by $M(M \geq J)$ analog subarrays, each having $N$ isotropic antenna elements with omni-directional radiation patterns to serve $J$ users. Denote the index sets of users and subarrays as $\mathcal{J}$ and $\mathcal{M}$, respectively. Specifically, each subarray with $N$ adjustable phase shifers is connected to a RF chain. At the output of each subarray, the signals are sampled via analog-to-digital (A/D) conversions at baseband followed 


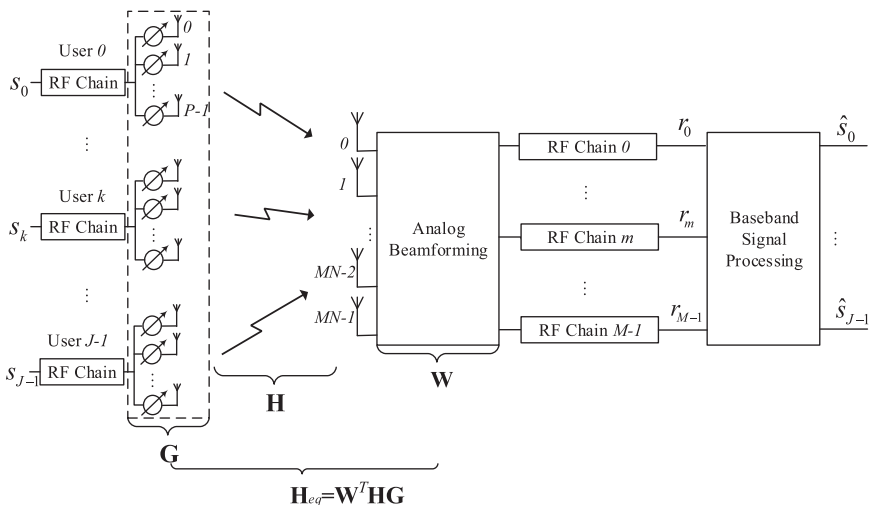

Fig. 1. Block diagram of mmWave multiuser receivers with analog BF implemented using phase shifters followed by RF chains and digital baseband signal processing.

by further digital signal processing. Each user is assumed to be equipped with a single analog array with $P$ antennas for lower hardware cost and power consumption. The hybrid array has two types of regular configurations: localized and interleaved arrays, as illustrated in Fig. 2 for an example of uniform planar array with $2 \times 2$ subarrays.

Denote the transmitted symbols and power of $J$ users as $\mathbf{s}=\left[s_{0}, s_{1}, \ldots, s_{J-1}\right]^{T}$, where $\mathbf{s} \sim \mathcal{C N}(\mathbf{0}, \mathbf{P})$ and $\mathbf{P}=\operatorname{diag}\left\{p_{0}, p_{1}, \ldots, p_{J-1}\right\}$, respectively. Denote the $\mathrm{BF}$ vector of user $k \in \mathcal{J}$ as $\mathbf{g}_{k} \in \mathbb{C}^{P \times 1}$, with each element having a constant amplitude $1 / \sqrt{P}$. Let $\mathbf{H}_{m}=$ $\left[\mathbf{H}_{m, 0}, \mathbf{H}_{m, 1}, \ldots, \mathbf{H}_{m, J-1}\right] \in \mathbb{C}^{N \times J P}$, where $\mathbf{H}_{m, k} \in \mathbb{C}^{N \times P}$ denotes the propagation channel between user $k$ and subarray $m$ of the receiver. Therefore, the received signal $\mathbf{y}_{m}$ at subarray $m$ can be expressed as

$$
\mathbf{y}_{m}=\sum_{k=0}^{J-1} \mathbf{H}_{m, k} \mathbf{g}_{k} s_{k}+\mathbf{n}_{m}=\mathbf{H}_{m} \mathbf{G s}+\mathbf{n}_{m},
$$

where $\mathbf{G} \in \mathbb{C}^{J P \times J}$ denotes the analog BF matrix at all users, as

$$
\mathbf{G}=\left(\begin{array}{cccc}
\mathbf{g}_{0} & \mathbf{0} & \cdots & \mathbf{0} \\
\mathbf{0} & \mathbf{g}_{1} & \cdots & \mathbf{0} \\
\vdots & \vdots & \ddots & \vdots \\
\mathbf{0} & \mathbf{0} & \cdots & \mathbf{g}_{J-1}
\end{array}\right),
$$

and $\mathbf{n}_{m} \sim \mathcal{C N}\left(\mathbf{0}, \sigma_{n}^{2} \mathbf{I}_{N}\right)$ denotes the additive white Gaussian noise (AWGN) at subarray $m$. By stacking $\mathbf{y}_{m}$ one by one, we get the received signal $\mathbf{y}=\left[\mathbf{y}_{0}^{T}, \mathbf{y}_{1}^{T}, \ldots, \mathbf{y}_{M-1}^{T}\right]^{T}$ as

$$
\mathbf{y}=\mathbf{H G s}+\mathbf{n},
$$

where $\mathbf{H}=\left[\mathbf{H}_{0}^{T}, \mathbf{H}_{1}^{T}, \ldots, \mathbf{H}_{M-1}^{T}\right]^{T} \in \mathbb{C}^{N M \times J P}$, and $\mathbf{n}=$ $\left[\mathbf{n}_{0}^{T}, \mathbf{n}_{1}^{T}, \ldots, \mathbf{n}_{M-1}^{T}\right]^{T}$. The detailed channel matrix will be described in Section II.B. Denote the analog BF matrix at the BS, $\mathbf{W} \in \mathbb{C}^{N M \times M}$, as

$$
\mathbf{W}=\left(\begin{array}{cccc}
\mathbf{w}_{0} & \mathbf{0} & \cdots & \mathbf{0} \\
\mathbf{0} & \mathbf{w}_{1} & \cdots & \mathbf{0} \\
\vdots & \vdots & \ddots & \vdots \\
\mathbf{0} & \mathbf{0} & \cdots & \mathbf{w}_{M-1}
\end{array}\right)
$$

where $\mathbf{w}_{m} \in \mathbb{C}^{N \times 1}$ denotes the weight vector of subarray $m$, each element with constant amplitude $1 / \sqrt{N}$. The signals

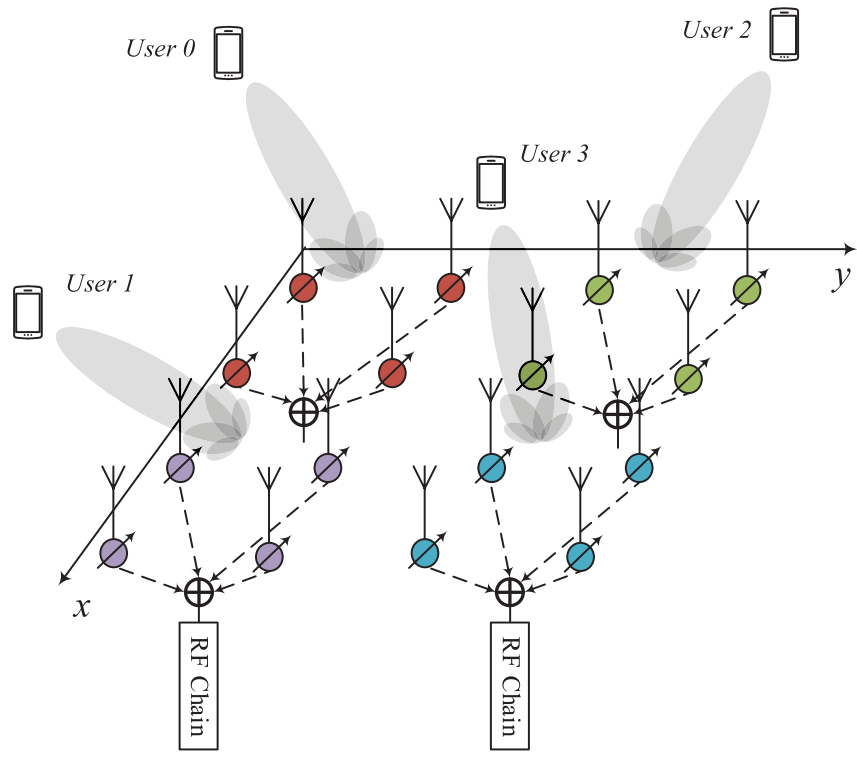

(a)

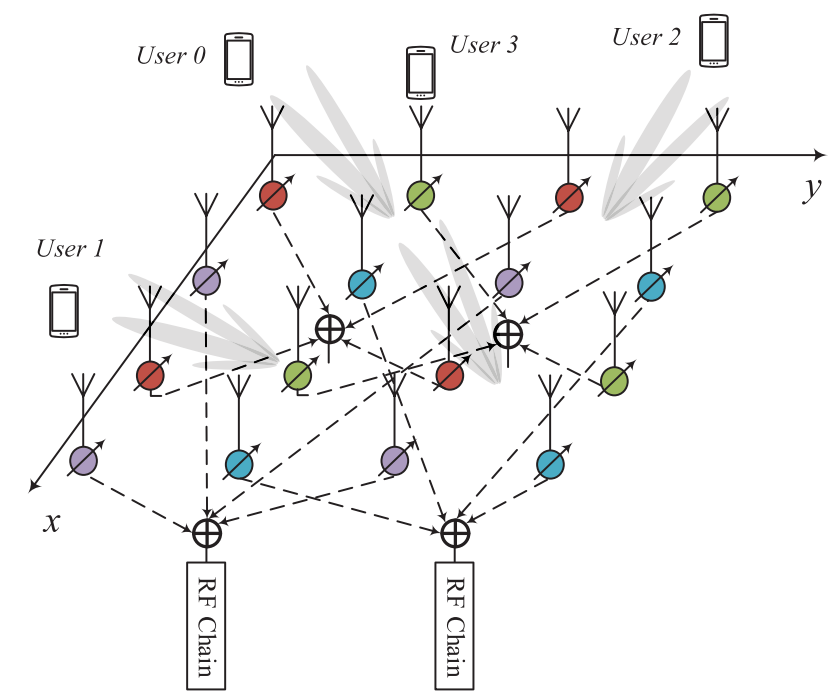

(b)

Fig. 2. An example of two typical partially-connected hybrid arrays with $2 \times 2$ subarrays: (a) Localized arrays, where the elements in each subarray are adjacent to each other. (b) Interleaved arrays, where the elements in each subarray are evenly distributed over the whole array.

from all the analog subarrays, $\mathbf{r}$, are given by

$$
\mathbf{r}=\mathbf{W}^{T} \mathbf{y}=\mathbf{W}^{T} \mathbf{H G} \mathbf{s}+\mathbf{W}^{T} \mathbf{n}=\mathbf{H}_{e q} \mathbf{s}+\mathbf{W}^{T} \mathbf{n},
$$

where $\mathbf{H}_{e q}=\left[\mathbf{h}_{e q, 0}, \mathbf{h}_{e q, 1}, \ldots, \mathbf{h}_{e q, J-1}\right] \in \mathbb{C}^{M \times J}$ denotes the equivalent digital baseband channel between the users and BS receiver, and includes the mmWave channels and the analog $\mathrm{BF}$ at the users and BS.

Assuming that CSI is perfectly known at the BS, the achievable uplink sum-rate is given by [21] and [22]

$$
\begin{aligned}
R & =\log _{2}\left|\mathbf{I}_{M}+\frac{1}{\sigma_{n}^{2}} \sum_{k=0}^{J-1} p_{k} \mathbf{h}_{e q, k} \mathbf{h}_{e q, k}^{H}\right| \\
& =\log _{2}\left|\mathbf{I}_{M}+\frac{1}{\sigma_{n}^{2}} \mathbf{H}_{e q} \mathbf{P} \mathbf{H}_{e q}^{H}\right| .
\end{aligned}
$$


Assuming that the transmission power from all users are the same, i.e., $p_{k}=p$, (4) becomes

$$
R=\log _{2}\left|\mathbf{I}_{J}+\frac{p}{\sigma_{n}^{2}} \sum_{m=0}^{M-1}\left(\mathbf{w}_{m}^{T} \mathbf{H}_{m} \mathbf{G}\right)^{H}\left(\mathbf{w}_{m}^{T} \mathbf{H}_{m} \mathbf{G}\right)\right|,
$$

which shows that the achievable uplink sum-rate for a partially-connected hybrid array depends on the SNR and the correlation between different subarrays' equivalent channels.

\section{B. Channel Model}

Compared to conventional microwave channels with rich scattering, mmWave channel models [12], [23] in the urban microcell environments can be characterized by the LOS component and non-LOS (NLOS) scattering components, where LOS path dominates the power distribution across the multipath. Therefore, Rician fading with a large Rician factor is typically applied to mmWave channel models.

Assume that the channel is frequency flat fading, time invariant for each slot, and may change from slot to slot. ${ }^{1}$ Here, we consider a uniform linear array for simplicity. Accordingly, $\mathbf{H}_{m, k}$ is assumed to be decomposed into a deterministic channel induced by LOS and a random channel induced by the scattering components [12] as

$$
\begin{aligned}
\mathbf{H}_{m, k}= & \sqrt{\frac{K_{k}}{1+K_{k}}} \mathbf{a}_{B S, m}\left(\theta_{k}\right) \mathbf{a}_{U}^{H}\left(\phi_{k}\right) \\
& +\sqrt{\frac{1}{L\left(1+K_{k}\right)}} \sum_{l=1}^{L} a_{k, l} \mathbf{a}_{B S, m}\left(\theta_{k, l}\right) \mathbf{a}_{U}^{H}\left(\phi_{k, l}\right),
\end{aligned}
$$

where $K_{k}$ is known as the Rician factor for the $k$ th user's channel. For simplicity, we assume that all users have the same Rician factor, i.e., $K_{k}=K, \forall k$; The variables $\theta_{k} \in$ $\left[-\frac{\pi}{2}, \frac{\pi}{2}\right]$, and $\phi_{k} \in\left[-\frac{\pi}{2}, \frac{\pi}{2}\right]$ are the angles of incidence in LOS direction at the arrays of the BS and user $k$, respectively; $L$ is the number of the NLOS paths. $a_{k, l} \sim \mathcal{C N}(0,1), \theta_{k, l}$ and $\phi_{k, l}$ are the complex gain and the angles of incidence of the $l$ th NLOS path at the arrays of the BS and user $k$, respectively; $\mathbf{a}_{B S, m}(\theta)$ and $\mathbf{a}_{U}(\phi)$ denote the corresponding antenna array response vectors at the $m$ th subarray of the BS and the user, respectively. $\mathbf{a}_{U}(\phi)$ is given by $\mathbf{a}_{U}(\phi)=$ $\left[1, \ldots, e^{j \frac{2 \pi}{\lambda}(P-1) d \sin \phi}\right]^{T}$, where $\lambda$ is the carrier wavelength and $d$ is the adjacent element spacing.

For localized and interleaved arrays at the BS, the locations of the $n$th element $(n=0,1, \ldots, N-1)$ in the $m$ th subarray $(m=0,1, \ldots, M-1)$ are respectively arranged as $X_{n, m}=(n+m N) d$ and $X_{n, m}=(n M+m) d$. The array response $\mathbf{a}_{B S, m}(\theta)$ can then be written as $\mathbf{a}_{B S, m}(\theta)=$ $\left[e^{j \frac{2 \pi}{\lambda} X_{0, m} \sin \theta}, \ldots, e^{j \frac{2 \pi}{\lambda} X_{N-1, m} \sin \theta}\right]^{T}$. When the phase shift of the $n$th element in a subarray, $\alpha_{n}$, is set to $-\frac{2 \pi}{\lambda} n d_{e} \sin \theta^{\prime}$, the normalized array factor of a subarray can be expressed as

$$
P_{s}(\theta)=\frac{\sin \left[N \frac{\pi}{\lambda} d_{e}\left(\sin \theta-\sin \theta^{\prime}\right)\right]}{\sqrt{N} \sin \left[\frac{\pi}{\lambda} d_{e}\left(\sin \theta-\sin \theta^{\prime}\right)\right]},
$$

\footnotetext{
${ }^{1}$ In this paper, the proposed schemes and channel estimation method can be potentially extended to wideband mmWave systems [24], where the proposed reference signals are applied to all subcarriers and frequency-domain AoA estimation [25] can be performed.
}

where $d_{e}=d$ for localized arrays, whereas $d_{e}=M d$ for interleaved arrays. Similarly, we have the array factor at the user given by

$$
P_{u}(\phi)=\frac{\sin \left[P \frac{\pi}{\lambda} d\left(\sin \phi-\sin \phi^{\prime}\right)\right]}{\sqrt{P} \sin \left[\frac{\pi}{\lambda} d\left(\sin \phi-\sin \phi^{\prime}\right)\right]},
$$

Note that individual subarrays in localized and interleaved arrays have different beam patterns due to different subarray apertures as shown in Fig. 2. The former has wider beamwidth without grating lobes and achieves larger LOS-MIMO capacity, while the latter has larger grating lobes with narrower beam and lower AoA estimation complexity [2]. With the same number of elements in a subarray, the difference of the beamwidth between them is due to different distances between adjacent elements, e.g., the localized arrays have smaller distance leading to wider beamwidth. However, in a single user system, both arrays have the same overall pattern when using the same digital BF [20]. For a multiuser system, the interferences between the subarray beams directing at different users have different properties. For example, due to wide beamwidth, the localized subarray will result in high interference among the users with close AoAs, while the interleaved subarray will lead to large disturbance when the beam directing at one user falls into the grating lobes of another user's beam. As a result, we need to use a digital equalizer to reduce the multiuser interference.

\section{User-Directed BEAMForming Schemes}

Given G, the sum-rate in (5) can be maximized by optimizing $\mathbf{W}$. However, the optimization of $\mathbf{W}$ requires the full CSI between each pair of antennas at the BS and each user, which substantially increases the complexity of channel estimation especially when massive hybrid array is used.

To simplify the analog BF structure and reduce the complexity of channel estimation, we propose three user-directed subarray allocation schemes that only require the knowledge of dominating $\mathrm{AOA}$ and equivalent channel information. The basic idea is to allocate subarrays to users one by one using an iterative cancellation approach. Once one subarray's BF vector is determined, its corresponding equivalent channel is removed from the equivalent channel matrix for determining the $\mathrm{BF}$ vectors of other subarrays. These schemes are applicable to both interleaved and localized arrays.

\section{A. Three User-Directed Per Subarray Based Schemes}

When the Rician factor, $K$, is sufficiently large and the AOA information is available, BF design at the BS based on the dominating AoA generally only selects a BF matrix, $\mathbf{W}$, to direct each subarray's beam towards a specific user. That is to say, $\mathbf{w}_{m}(m \in \mathcal{M})$ is designed by selecting one of $\mathbf{a}_{B S, 0}^{*}\left(\theta_{k}\right) / \sqrt{N}(k \in \mathcal{J})$. Similarly, if each user knows its angle of departure (AoD), it is possible to design its BF vector based on its dominating AoD. Since there is no cooperation among users, all users may steer their beams towards the receiver, i.e., $\mathbf{g}_{k}=\mathbf{a}_{U}\left(\phi_{k}\right) / \sqrt{P}$. As proved in [8], the optimal eigen-BF vectors for the channel in (6) converge to the antenna 


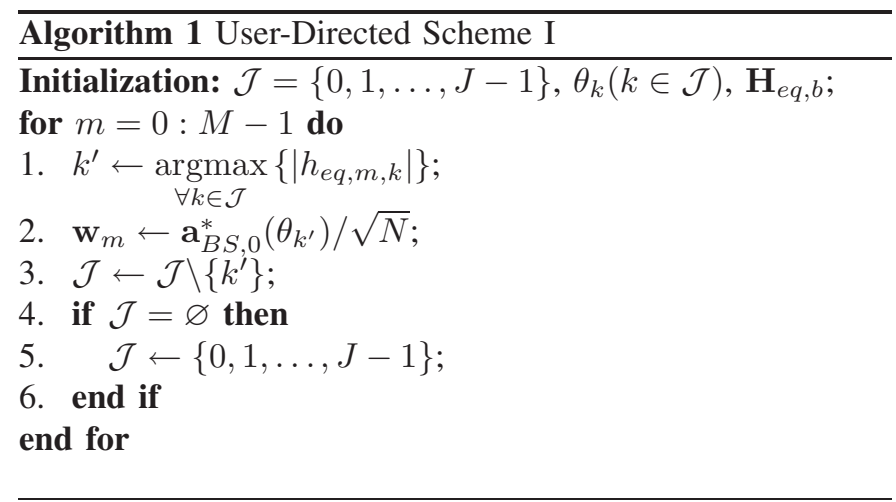

array response vectors approximately in the LOS directions for a large array.

We define $h_{e q, m, k} \triangleq \mathbf{a}_{B S, 0}^{H}\left(\theta_{k}\right) \mathbf{H}_{m, k} \mathbf{a}_{U}\left(\phi_{k}\right)$ as the equivalent channel between user $k$ and subarray $m$. Therefore, the equivalent channel matrix between all users and subarrays can be constructed as

$$
\mathbf{H}_{e q, b}=\left(\begin{array}{cccc}
h_{e q, 0,0} & h_{e q, 0,1} & \cdots & h_{e q, 0, J-1} \\
h_{e q, 1,0} & h_{e q, 1,1} & \cdots & h_{e q, 1, J-1} \\
\vdots & \vdots & \ddots & \vdots \\
h_{e q, M-1,0} & h_{e q, M-1,1} & \cdots & h_{e q, M-1, J-1}
\end{array}\right) .
$$

If $\mathbf{H}_{e q, b}$ is available at the BS, the allocation of subarrays to users can be optimized to jointly exploit the directivity and the spatial diversity. To seek the fairness among different users, we assume that every $J$ allocations guarantee $J$ different users to be allocated with different subarrays. ${ }^{2}$ This will lead to multiple subarrays' beams being steered towards a specific user and thus achieve higher antenna gain and diversity gain.

We propose three user-directed subarray allocation schemes, where the allocation process can be implemented through optimization in either one- (along row/column) or two-dimension (across both row and column) in $\mathbf{H}_{e q, b}$. In one-dimension optimization, scheme I seeks to find a subarray for the right user, while scheme II seeks to find a user for the right subarray. In two-dimension optimization, scheme III tries to find the right subarray for the right user in each iteration. These schemes attempt to reduce channel correlation while allowing optimization following certain criterions. They are summarized in Algorithm I, II and III respectively, where $\mathcal{J} \backslash\left\{k^{\prime}\right\}$ denotes the complement of $\left\{k^{\prime}\right\}, \varnothing$ denotes the empty set and $\bmod \{\cdot, \cdot\}$ stands for the modulo operation.

After the BF vectors of all subarrays are determined using the above schemes, the equivalent digital baseband channel, $\mathbf{H}_{e q, a}$, is obtained, so is the achievable sum-rate, where the subscript of " $a$ " is used to distinguish $\mathbf{H}_{e q, b}$.

\section{B. SIC Schemes}

Here, we also derive an SIC solution following that in [16] as the near-optimal benchmark for comparison. The basic idea behind the SIC scheme is to decompose the achievable

\footnotetext{
${ }^{2}$ When $\bmod \{M, J\} \neq 0$, i.e., the number of subarrays allocated to some users is one less than the others', the system can employ round-robin scheduling in different data frames to seek the fairness among users.
}
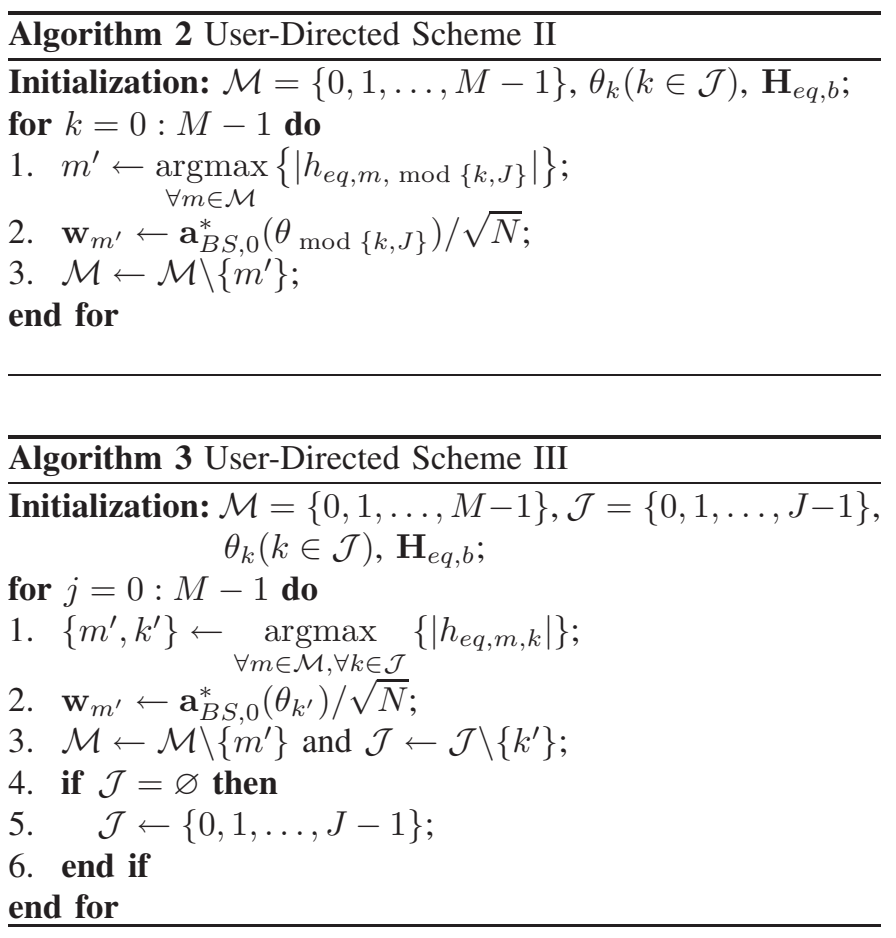

sum-rate optimization problem into a series of subarray based subrate optimization problems, and to iteratively optimize $\mathbf{w}_{m}$ from the first to the last by applying the principle of SIC for multiuser signal detection. From (5), the corresponding achievable sum-rate can be derived as

$R=\sum_{m=0}^{M-1} \log _{2}\left(1+\frac{p}{\sigma_{n}^{2}} \mathbf{w}_{m}^{T} \mathbf{H}_{m} \mathbf{G} \mathbf{Q}_{m-1}^{-1}\left(\mathbf{w}_{m}^{T} \mathbf{H}_{m} \mathbf{G}\right)^{H}\right)$,

where $\mathbf{Q}_{m}=\mathbf{I}_{J}+\frac{p}{\sigma_{n}^{2}} \sum_{m^{\prime}=0}^{m}\left(\mathbf{w}_{m^{\prime}}^{T} \mathbf{H}_{m^{\prime}} \mathbf{G}\right)^{H}\left(\mathbf{w}_{m^{\prime}}^{T} \mathbf{H}_{m^{\prime}} \mathbf{G}\right), 0 \leq$ $m \leq M-2$, and $\mathbf{Q}_{-1}=\mathbf{I}_{J}$. Eq. (7) holds using the matrix determinant lemma $\left|\mathbf{A}+\mathbf{u v}^{H}\right|=\left(1+\mathbf{v}^{H} \mathbf{A}^{-1} \mathbf{u}\right)|\mathbf{A}|$, where A is invertible.

To maximize the achievable rate for each subarray, i.e., $\log _{2}\left(1+\frac{p}{\sigma_{n}^{2}} \mathbf{w}_{m}^{T} \mathbf{H}_{m} \mathbf{G} \mathbf{Q}_{m-1}^{-1} \mathbf{G}^{H} \mathbf{H}_{m}^{H} \mathbf{w}_{m}^{*}\right)$, we need to perform the singular value decomposition (SVD) of the Hermitian matrix $\mathbf{H}_{m} \mathbf{G Q}_{m-1}^{-1} \mathbf{G}^{H} \mathbf{H}_{m}^{H}$ from $m=0$ to $M-1$. The conjugate of the singular vector corresponding to the maximal singular value, $\mathbf{v}_{o}^{*}$, is thus the optimal unconstrained $\mathbf{w}_{m}$. Here, we name it as the benchmarking scheme $(a)$. This is equivalent to seeking optimal $\mathbf{w}_{m}$ by considering the impact from the first $m-1$ subarrays, while not from the remaining $M-m$ subarrays. When the $n$th element of $\mathbf{w}_{m}$ needs to meet the constant amplitude constraint, a feasible solution is given by $\mathbf{w}_{m}=\frac{1}{\sqrt{N}} e^{-j \angle\left(\mathbf{v}_{o}\right)}$, where $\angle(\mathbf{a})$ denotes element-wise phase angle operation for $\mathbf{a}$. This solution is referred to as the benchmarking scheme $(b)$.

Note that the computational complexity of SIC based optimal solution is quite high since it requires SVD and matrix inversion calculations. Although a low-complexity algorithm in [16] was proposed to avoid such calculations, all users' CSI at each antenna element is still needed, which is unrealistic for a large hybrid array. Also when the received SNR changes, 
the optimal solution of $\mathbf{w}_{m}$ needs to be updated. The acquisition of CSI at each antenna element inevitably leads to high complexity. On the other hand, since the proposed schemes only require $J$ AoAs and $M J$ equivalent channel information instead of $M N J$ full CSI, the implementation complexity of analog $\mathrm{BF}$ and channel estimation will be significantly reduced. Also, subarray allocation is performed basically using the information in the RF domain, which reduces the signal processing complexity and power consumption.

\section{Channel Estimation Method And REFERENCE SIGNALS DESIGN}

Due to the constraints on the number of RF chains and ana$\log \mathrm{BF}$, conventional pilot-aided channel estimation methods for fully digital systems are not suitable for hybrid systems. In this section, we propose a novel channel estimation method for hybrid mmWave multiuser MIMO systems, which includes three steps as shown in Algorithm IV, and is detailed next.

In the first step, we estimate the strongest AoAs at the users by searching the beamsteering codebooks, which will be exploited to determine the analog BF matrix G. In the second step, we propose a long reference signal design for interleaved arrays to estimate the strongest AoAs at the BS and $\mathbf{H}_{e q, b}$, where the AOA estimation problem for multiple users is converted to multiple single user AOA estimation problems, so that a simple DBT AOA estimation scheme can be applied. We allocate the subarrays to users following the proposed user-directed schemes in Section III. In the third step, the users transmit the designed short reference signals to facilitate the estimation of $\mathbf{H}_{e q, a}$, which is then used for multiuser detection. For localized arrays, the design can be extended to the generalized DBT [20]. However, due to the required extra searching for AoAs, the reference signals will be longer than that for the interleaved array.

\section{A. Details of Proposed Channel Estimation Method}

In Step 1 of Algorithm IV, the BS transmits a training sequence by using one of the omni-directional antennas in hybrid arrays, to facilitate the users to find the strongest AoAs. Due to the use of only one RF chain and the limited processing capability at the user, we adopt beam-searching based approach in several predefined directions for AoA estimation. Since phase shifters are digitally implemented, $\mathrm{BF}$ angles may be chosen from finite-size codebook [11]. Specifically, in this paper, we use the beamsteering codebook for users' BF design, which has the same form as $\mathbf{a}_{U}(\phi)$, to simplify the codebook design due to single parameter quantization. Denote $\mathcal{F}$ as the beamsteering codebook with cardinality $|\mathcal{F}|=V$, and $\mathbf{a}_{U}\left(-\frac{\pi}{2}+\frac{\pi v}{V}\right) / \sqrt{P}(v=0,1, \ldots, V-1)$ as the beamsteering vectors in $\mathcal{F}$.

When $\mathbf{a}_{U}\left(-\frac{\pi}{2}+\frac{\pi v}{V}\right) / \sqrt{P}$ is used as the BF vector of user $k$, let $\left|r_{k, v}^{U}\right|$ represent the corresponding received $\mathrm{BF}$ signal amplitude. Therefore, the transmit BF vector for user $k, \widehat{\mathrm{g}}_{k}$, can be selected from $\mathcal{F}$ such that

$$
\widehat{\mathrm{g}}_{k}=\underset{\forall \mathbf{a}_{U}\left(-\frac{\pi}{2}+\frac{\pi v}{V}\right) / \sqrt{P} \in \mathcal{F}}{\operatorname{argmax}}\left\{\left|r_{k, v}^{U}\right|\right\} .
$$

Alternatively, the selection process can be implemented by searching the codebook with efficient beam training algorithms developed in [26].

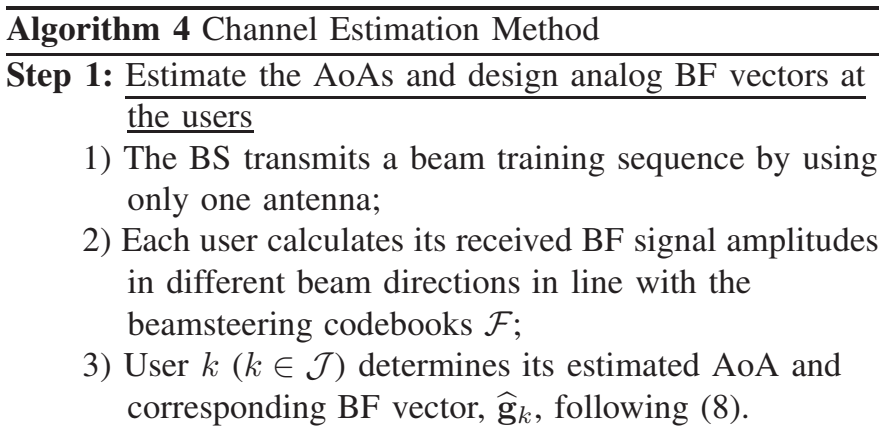

Step 2: Estimate the AoAs and design analog BF matrix at the BS

4) User $k(k \in \mathcal{J})$ transmits its allocated long reference signals by using $\widehat{\mathrm{g}}_{k}$ as BF vector;

5) The BS receiver estimates all users' AoAs using the DBT algorithm shown in Algorithm V;

6) The BS receiver calculates the received BF signal amplitude $\left|r_{m, k}^{B S}\right|, \forall m \in \mathcal{M}, \forall k \in \mathcal{J}$ in the estimated AoA directions;

7) The BS determines the allocation of subarrays to users and resulting BF matrix $\widehat{\mathbf{W}}$ following the proposed user-directed schemes, where $\left|h_{e q, m, k}\right|$ is replaced by $\left|r_{m, k}^{B S}\right|$.

Step 3: Estimate the equivalent baseband channel and design the ZF equalizer

8) User $k(k \in \mathcal{J})$ transmits its allocated short reference signals by using $\widehat{\mathrm{g}}_{k}$ as $\mathrm{BF}$ vector and the BS receives these signals by using $\widehat{\mathrm{W}}$ as $\mathrm{BF}$ matrix;

9) The BS receiver estimates all users' equivalent baseband channel, $\widehat{\mathbf{H}}_{e q, a}$;

10) The $B S$ receiver determines the $Z F$ equalizer as $\left(\widehat{\mathbf{H}}_{e q, a}^{H} \widehat{\mathbf{H}}_{e q, a}\right)^{-1} \widehat{\mathbf{H}}_{e q, a}^{H}$.

In Step 2, each user transmits its allocated long reference signals by leveraging BF vector $\widehat{\mathrm{g}}_{k}$ obtained in Step 1, which are assumed to be synchronously received by the BS receiver. The synchronization can be achieved via the control information included in downlink control packets. All users follow the allocated time slots to transmit their reference signals. When the signals are received, each analog subarray adjusts their phases at each antenna element using analog phase shifters, and then combines the signals with an analog mixer and downconverts to generate a complex baseband signal. Following the outputs of analog beamformers and A/D converters, uplink AoA estimation using DBT is performed and the phase shifts at the subarrays are adjusted via a feedback path. The AoA estimation process is recursively carried out until the latest AoA estimates for all users are converged within a tolerable range. Please refer to Section IV.B and $\mathrm{C}$ for details of DBT based AoA estimation and long reference signal design.

When the AoA estimates, $\widehat{\theta}_{k}(k \in \mathcal{J})$, are determined and the corresponding beamsteering vectors, $\mathbf{a}_{B S, 0}^{*}\left(\widehat{\theta}_{k}\right) / \sqrt{N}$, are set to $\mathrm{BF}$ vectors, $\widehat{\mathbf{w}}_{m}(m \in \mathcal{M})$, the received BF signal, $r_{m, k}^{B S}$, is given by

$r_{m, k}^{B S}=\widehat{\mathbf{w}}_{m}^{T} \mathbf{H}_{m, k} \widehat{\mathbf{g}}_{k} s_{k}+\widehat{\mathbf{w}}_{m}^{T} \mathbf{n}_{m}=h_{e q, m, k} s_{k}+\widehat{\mathbf{w}}_{m}^{T} \mathbf{n}_{m}$. 
Since all users have the same transmission power and $\widehat{\mathbf{w}}_{m}^{T} \mathbf{n}_{m} \sim \mathcal{C N}\left(0, \sigma_{n}^{2}\right), \forall m \in \mathcal{M}$, we replace $\left|h_{e q, m, k}\right|$ with $\left|r_{m, k}^{B S}\right|$ in Algorithms I-III to perform the allocation of subarrays and determine the BF matrix $\widehat{\mathbf{W}}$.

In Step 3, each user transmits its allocated short reference signals by using $\widehat{\mathrm{g}}_{k}$ obtained in Step 1 . Then, the BS receives these signals and applies $\widehat{\mathbf{W}}$ obtained in Step 2. This will facilitate the equivalent baseband channel estimation. Denote the short reference signal for the $k$ th user as $\mathbf{s}_{k}^{s r}=$ $\left[s_{k}^{s r}[0], s_{k}^{s r}[1], \ldots, s_{k}^{s r}[J-1]\right]^{T}$. Thus, all users' reference signals are stacked in a matrix, $\mathbf{S}=\left[\mathbf{s}_{0}^{s r}, \mathbf{s}_{1}^{s r}, \ldots, \mathbf{s}_{J-1}^{s r}\right]^{T} \in$ $\mathbb{C}^{J \times J}$. If $\mathbf{S}$ is designed to satisfy the condition of $\mathbf{S S}^{H}=$ $J p \mathbf{I}_{J}$, i.e., $\mathbf{s}_{k}^{s r H} \mathbf{s}_{k}^{s r}=J p$ and $\mathbf{s}_{k}^{s r H} \mathbf{s}_{j}^{s r}=0, \forall k, j=$ $0, \ldots, J-1$ and $k \neq j, \mathbf{H}_{e q, a}$ can be estimated using the LS method by

$$
\widehat{\mathbf{H}}_{e q, a}=\frac{1}{J p} \mathbf{R S}^{H}=\mathbf{H}_{e q}+\frac{1}{J p} \widehat{\mathbf{W}}^{T} \mathbf{N S}^{H},
$$

where $\mathbf{R} \in \mathbb{C}^{M \times J}$ denotes the received BF signals, and $\mathbf{N} \sim \mathcal{C N}\left(\mathbf{0}, J \sigma_{n}^{2} \mathbf{I}_{M N}\right)$ denotes the AWGN matrix. Therefore, the MSE of $\widehat{\mathbf{H}}_{e q, a}$ can be evaluated by

$$
\begin{aligned}
\operatorname{MSE}_{\widehat{\mathbf{H}}_{e q, a}} & =\frac{1}{M J} \mathbb{E}\left[\left\|\widehat{\mathbf{H}}_{e q, a}-\mathbf{H}_{e q, a}\right\|_{F}^{2}\right] \\
& =\frac{\sigma_{n}^{2} \operatorname{Tr}\left(\widehat{\mathbf{W}}^{H} \widehat{\mathbf{W}}\right)}{M J p}=\frac{\sigma_{n}^{2}}{J p} .
\end{aligned}
$$

It is observed that the MSE is inversely proportional to the average power and the length of the reference signals.

The reference signals in Step 2 are used to recursively update the receive $\mathrm{BF}$ vectors at the $\mathrm{BS}$ to improve uplink AoA estimation, and thus it may need a comparatively long signal period. On the other hand, the reference signals in Step 3 are relatively short due to no need for recursive estimation. However, the total period of the reference signals is assumed to be smaller than the coherence time of the channel in this paper. Based on $\widehat{\mathbf{H}}_{e q, a}$, the $\mathrm{ZF}$ equalizer is determined as $\left(\widehat{\mathbf{H}}_{e q, a}^{H} \widehat{\mathbf{H}}_{e q, a}\right)^{-1} \widehat{\mathbf{H}}_{e q, a}^{H}$ for multiuser detection.

\section{B. Signal Model and DBT Algorithm for AoA Estimation}

Here, we introduce the DBT algorithm for AoA estimation. First, assume that there is only one user (e.g., the $k$ th user) in the system. Denote the $q$ th symbol of its long reference signal with constant envelope as $s_{k}^{l r}[q]$, and the received $\mathrm{BF}$ signal at the $m$ th subarray as $r_{m, k}^{l r}[q]$, where $q=1,2, \ldots, Q$ and $Q$ is the length of long reference signals. We assume the phase shifters taking values of, $\alpha_{0}, \alpha_{1}, \ldots, \alpha_{N-1}$, in all the subarrays, which greatly simplifies the analog implementation due to single configuration. Therefore, $r_{m, k}^{l r}[q]$ is given by

$$
\begin{aligned}
r_{m, k}^{l r}[q]= & {\left[\sqrt{\frac{K}{1+K}} P_{s}\left(\theta_{k}\right) P_{u}\left(\phi_{k}\right) e^{j \frac{2 \pi}{\lambda} m d \sin \theta_{k}}\right.} \\
& +\sqrt{\frac{1}{L(1+K)} \sum_{l=1}^{L} a_{k, l} P_{s}\left(\theta_{k, l}\right) P_{u}\left(\phi_{k, l}\right)} \\
& \left.\cdot e^{j \frac{2 \pi}{\lambda} m d \sin \theta_{k, l}}\right] s_{k}^{l r}[q]+n_{m}[q] .
\end{aligned}
$$

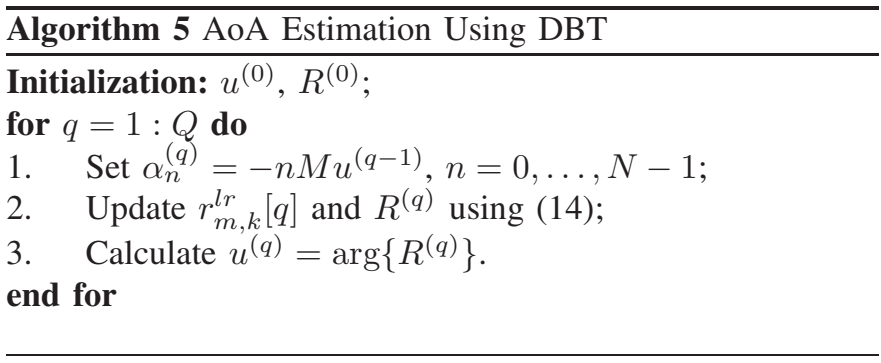

where $n_{m}[q]$ denotes the noise at the output of the $m$ th subarray. It is observed from (12) that, the LOS AoA information can be estimated from the phase difference between the adjacent subarray output signals, provided that $P_{s}\left(\theta_{k}\right) P_{u}\left(\phi_{k}\right) \neq 0$ and the interference induced by NLOS is not high. In addition, the estimated AoA can be fed back to adjust the phase shifting values in order to align the beam. Thus, the enhanced BF signal contributes to the following AoA estimate, i.e., the AoA estimation is recursive. This is known as the DBT algorithm [20] summarized in Algorithm V.

Let the cross-correlation of any two adjacent subarray output signals be $R$. Assuming that the NLOS path gains and noise components are independent, we have

$$
\begin{aligned}
R= & \mathbb{E}\left[r_{m, k}^{l r *}[q] r_{m+1, k}^{l r}[q]\right] \\
= & \underbrace{\frac{p K}{1+K}\left|P_{s}\left(\theta_{k}\right) P_{u}\left(\phi_{k}\right)\right|^{2} e^{j \frac{2 \pi}{\lambda} d \sin \theta_{k}}}_{\text {desired component }} \\
& +\underbrace{\frac{p}{L(1+K)} \sum_{l=1}^{L}\left|P_{s}\left(\theta_{k, l}\right) P_{u}\left(\phi_{k, l}\right)\right|^{2} e^{j \frac{2 \pi}{\lambda} d \sin \theta_{k, l}}}_{\text {NLOS interference }} .
\end{aligned}
$$

It is seen that the LOS AoA information is contained in the argument of the desired component. Let $u=\frac{2 \pi}{\lambda} d \sin \left(\theta_{k}\right)$, which falls in the range $[-\pi, \pi)$ assuming $d \leq \frac{\lambda}{2}$. If the NLOS interference is ignored in (13), we have $u=\arg \{R\}$. Using adaptive filtering theory, the cross-correlation evaluated based on the first $i$ received symbols, $R^{(q)}$, can be represented as a weighted sum of that on the first $q-1$ symbols, $R^{(q-1)}$, and an instaneous differential signal, $r_{m, k}^{l r *}[q] r_{m+1, k}^{l r}[q]$, as

$$
R^{(q)}=(1-\mu) R^{(q-1)}+\mu \sum_{m=0}^{M-2} r_{m, k}^{l r *}[q] r_{m+1, k}^{l r}[q],
$$

where $0<\mu<1$ is the updating coefficient.

\section{Design for Multiuser Reference Signals}

Assume that there are $J$ users in the system. Denote $r_{m}^{l r}[q]$ as the sum of $r_{m, k}^{l r}[q]$ in $(12), k=0,1, \ldots, J-1$. By iteratively substituting $\left\{R^{(q-1)}, R^{(q-2)}, \ldots, R^{(0)}\right\}$ into (14), it is rewritten as

$$
R^{(q)}=(1-\mu)^{q} R^{(0)}+\mu \sum_{m=0}^{M-2} \sum_{i=1}^{q}(1-\mu)^{q-i} r_{m}^{l r *}[i] r_{m+1}^{l r}[i] .
$$




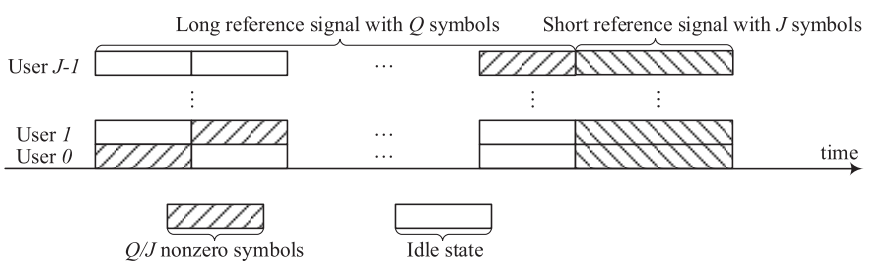

Fig. 3. Reference signal design along time slots.

Ignoring $R^{(0)}$ and the noise impact for simplicity, we can write $R^{(q)}$, for the desired user $k$, as

$$
\begin{aligned}
R_{k}^{(q)}= & \mu \sum_{m=0}^{M-2} \sum_{i=1}^{q}(1-\mu)^{q-i} \\
& \cdot(\underbrace{\mathbf{w}_{(i)}^{H} \mathbf{H}_{m, k}^{*} \widehat{\mathbf{g}}_{k}^{*} \mathbf{w}_{(i)}^{T} \mathbf{H}_{m+1, k} \widehat{\mathbf{g}}_{k}\left|s_{k}^{l r}[i]\right|^{2}}_{\text {desired component }} \\
& +\sum_{j \neq k} \mathbf{w}_{(i)}^{H} \mathbf{H}_{m, j}^{*} \widehat{\mathbf{g}}_{j}^{*} \mathbf{w}_{(i)}^{T} \mathbf{H}_{m+1, j} \widehat{\mathbf{g}}_{j}\left|s_{j}^{l r}[i]\right|^{2} \\
& \left.+\sum_{j} \sum_{j^{\prime} \neq j} \mathbf{w}_{(i)}^{H} \mathbf{H}_{m, j}^{*} \widehat{\mathbf{g}}_{j}^{*} \mathbf{w}_{(i)}^{T} \mathbf{H}_{m+1, j^{\prime}} \widehat{\mathbf{g}}_{j^{\prime}} s_{j}^{l r *}[i] s_{j^{\prime}}^{l r}[i]^{\prime}\right),
\end{aligned}
$$

where $\mathbf{w}_{(i)}$ denotes the BF vector in each subarray at the $i$ th received signal. Eq. (16) includes the desired component for user $k$, and the interference from other users consisting of two terms following the desired component. To eliminate the interference, we let $s_{j}^{l r}[i]=0, \forall j \neq k$ and $i=1, \ldots, q$. Let the set $\Pi_{k}(k \in \mathcal{J})$ represent the indices of the nonzero symbols allocated to the $k$ th user's reference signal. In order to estimate all users' AoAs during one reference signal period, we design $\Pi_{k}$ to satisfy the following conditions: 1) $\Pi_{k} \cap \Pi_{j}=\varnothing, \forall k \neq j$, and 2) $\bigcup_{k=0}^{J-1} \Pi_{k}=\mathbb{Q}$, where $\mathbb{Q}$ denotes universal set, i.e., $\mathbb{Q}=\{1, \ldots, Q\}$.

Without loss of generality, a feasible reference signal for any user $k$ can be given by

$$
\left|s_{k}^{l r}[q]\right|^{2}= \begin{cases}J p, & q \in \Pi_{k} \\ 0, & \text { otherwise }\end{cases}
$$

where $\Pi_{k}=\left\{\frac{Q}{J} k+1, \frac{Q}{J} k+2, \ldots, \frac{Q}{J}(k+1)\right\}$, and $\frac{Q}{J}$ is assumed to be an integer. Using (17) and the DBT algorithm described in Algorithm V, the BS receiver can estimate all users' AoAs.

The reference signal design along time slots is illustrated in Fig. 3. An example for two users is as follows. The first user transmits its nonzero reference signals, $s_{0}^{l r}[q]$, at $q=1, \ldots, \frac{Q}{2}$, while the other is in idle state. When the other user transmits nonzero $s_{1}^{l r}[q]$, at $q=\frac{Q}{2}+1$, $\ldots, Q$, the first user stays in idle state. The BS receiver uses the first $\frac{Q}{2}$ received signals to iteratively estimate the AoA for the first user, and the following $\frac{Q}{2}$ received signals for the other user's AoA estimation. Note that the proposed reference signal design not only guarantees that the average transmit power during one reference signal period remains unchanged for each user, but also effectively removes the multiuser interference for AoA estimation. It is shown from (17) that, multiple clusters of nonzero symbols need to be transmitted from multiple users during one reference signal period. Therefore, it requires the reference signal to be synchronized among different users. It is observed that the AoA is estimated and the phase shifting values are updated for every nonzero symbol, i.e., there are $\frac{Q}{J}$ recursive AoA updates in one reference signal period for each user. Since every AoA estimation is only for one specific user, the $\mathrm{BF}$ vectors for all subarrays can be set to the same at each iteration in order to improve AoA estimation SNR by combining cross-correlations from all adjacent subarray outputs.

\section{Performance Characterization FOR AoA ESTIMATION}

In this section, we employ the MSEs of the estimated $u$ to evaluate the performance of AoA estimation for the proposed reference signal design. Recursive MSE lower bounds in typical mmWave channels will be provided for comparison.

For illustration convenience, we consider a linear hybrid array of two subarrays. Let $\Delta u_{k}^{(q)}=u_{k}-u_{k}^{(q)}$, where $u_{k}^{(q)}$ is the estimate of $u_{k}$ using the $q$ th reference symbol. Hence, the equivalent array factor of a subarray can be denoted as $P_{s}\left(\Delta u_{k}^{(q-1)}\right)=\frac{\sin \left(N \Delta u_{k}^{(q-1)}\right)}{\sqrt{N} \sin \left(\Delta u_{k}^{(q-1)}\right)}$, which is a periodic function of $\Delta u_{k}^{(q-1)}$ with period $2 \pi$, and satisfies $\left|P_{s}\left(\Delta u_{k}^{(q-1)}\right)\right| \leq$ $\sqrt{N}$ and $\left|P_{s}(0)\right|=\sqrt{N}$. The $q$ th received signals for the two subarrays are respectively expressed as

$$
\begin{array}{r}
r_{0}^{l r}[q]=s_{k}^{l r}[q]\left(\sqrt{\frac{K}{1+K}} P_{s}\left(\Delta u_{k}^{(q-1)}\right) P_{u}\left(\phi_{k}\right)+\sqrt{\frac{1}{L(1+K)}}\right. \\
\left.\cdot \sum_{l=1}^{L} a_{k, l} P_{s}\left(\Delta u_{k, l}^{(q-1)}\right) P_{u}\left(\phi_{k, l}\right)\right)+n_{0}[q]
\end{array}
$$

and

$$
\begin{aligned}
r_{1}^{l r}[q]= & s_{k}^{l r}[q]\left(\sqrt{\frac{K}{1+K}} P_{s}\left(\Delta u_{k}^{(q-1)}\right) P_{u}\left(\phi_{k}\right) e^{j u_{k}}\right. \\
& \left.+\sqrt{\frac{1}{L(1+K)}} \cdot \sum_{l=1}^{L} a_{k, l} P_{s}\left(\Delta u_{k, l}^{(q-1)}\right) P_{u}\left(\phi_{k, l}\right) e^{j u_{k, l}}\right) \\
& +n_{1}[q] .
\end{aligned}
$$

The differential signal between $r_{0}^{l r}[q]$ and $r_{1}^{l r}[q]$ is given by (19), where $n[q]$ can be approximated as a complex Gaussian variable with zero mean and variance $\frac{2 J p K}{1+K}\left|P_{s}\left(\Delta u_{k}^{(q-1)}\right) P_{u}\left(\phi_{k}\right)\right|^{2}\left(\frac{J p}{L(1+K)} \sum_{l=1}^{L} \mid P_{s}\left(\Delta u_{k, l}^{(q-1)}\right)\right.$

$\left.\left.P_{u}\left(\phi_{k, l}\right)\right|^{2}+\sigma_{n}^{2}\right)$ by omitting higher order noise terms. It is seen from (19) that, the estimation of $u_{k}$ is formulated as the phase estimation of $\rho_{k}[q]$ based on the observed $\rho_{k}[i]$, $i=1, \ldots, q$, in the presence of previously estimated $u_{k}^{(i-1)}$, i.e., $u_{k}^{(q)}=\arg \left\{R_{k}^{(q)}\right\}=\arg \left\{\sum_{i=1}^{q} \rho_{k}[i]\right\}$. 


$$
\begin{aligned}
\rho_{k}[q]= & \frac{J p K}{1+K}\left|P_{s}\left(\Delta u_{k}^{(q-1)}\right) P_{u}\left(\phi_{k}\right)\right|^{2} e^{j u_{k}}+\frac{1}{\sqrt{L(1+K)}} \sum_{l=1}^{L} P_{s}\left(\Delta u_{k, l}^{(q-1)}\right) P_{u}\left(\phi_{k, l}\right)\left(s_{k}^{l r *}[q] a_{k, l}^{*} n_{1}[q]+s_{k}^{l r}[q] a_{k, l} n_{0}^{*}[q] e^{j u_{k, l}}\right) \\
& +\frac{J p}{1+K} \sqrt{\frac{K}{L}} P_{s}\left(\Delta u_{k}^{(q-1)}\right) P_{u}\left(\phi_{k}\right) \sum_{l=1}^{L} P_{s}\left(\Delta u_{k, l}^{(q-1)}\right) P_{u}\left(\phi_{k, l}\right)\left(a_{k, l} e^{j u_{k, l}}+a_{k, l}^{*} e^{j u_{k}}\right)+n_{0}^{*}[q] n_{1}[q] \\
& +\frac{J p}{L(1+K)} \sum_{l=1}^{L} \sum_{l^{\prime}=1}^{L} a_{k, l}^{*} P_{s}^{*}\left(\Delta u_{k, l}^{(q-1)}\right) P_{u}^{*}\left(\phi_{k, l}\right) a_{k, l^{\prime}} P_{s}\left(\Delta u_{k, l^{\prime}}^{(q-1)}\right) P_{u}\left(\phi_{k, l^{\prime}}\right) e^{j u_{k, l^{\prime}}} \\
& +\sqrt{\frac{K}{1+K}} P_{s}\left(\Delta u_{k}^{(q-1)}\right) P_{u}\left(\phi_{k}\right)\left(s_{k}^{l r *}[q] n_{1}[q]+s_{k}^{l r}[q] n_{0}^{*}[q] e^{j u_{k}}\right)=\frac{J p K}{1+K}\left|P_{s}\left(\Delta u_{k}^{(q-1)}\right) P_{u}\left(\phi_{k}\right)\right|^{2} e^{j u_{k}}+n[q],
\end{aligned}
$$

Since $R_{k}^{(q)}$ follows complex Gaussian distribution from (19), its joint conditional probability density function (pdf) for the given $u_{k}^{(i-1)}, i=1, \ldots, q$, denoted as $\mathbf{u}_{k}$, is given by [27]

$$
f_{R_{k}^{(q)} \mid \mathbf{u}_{k}}\left(R_{k}^{(q)} \mid \mathbf{u}_{k}\right)=\frac{1}{2 \pi \sigma_{R_{k}^{(q)}}^{2}} \exp \left(\frac{\left|R_{k}^{(q)}-m_{R_{k}^{(q)}}\right|^{2}}{2 \sigma_{R_{k}^{(q)}}^{2}}\right),
$$

where

$$
m_{R_{k}^{(q)}}=\frac{J p K}{1+K}\left|P_{u}\left(\phi_{k}\right)\right|^{2} \sum_{i=1}^{q}\left|P_{s}\left(\Delta u_{k}^{(i-1)}\right)\right|^{2} e^{j u_{k}}
$$

and

$$
\begin{aligned}
\sigma_{R_{k}^{(q)}}^{2}= & \frac{J p K}{1+K}\left|P_{u}\left(\phi_{k}\right)\right|^{2} \sum_{i=1}^{q}\left|P_{s}\left(\Delta u_{k}^{(i-1)}\right)\right|^{2} \\
& \cdot\left(\frac{J p}{L(1+K)} \sum_{l=1}^{L}\left|P_{s}\left(\Delta u_{k, l}^{(i-1)}\right) P_{u}\left(\phi_{k, l}\right)\right|^{2}+\sigma_{n}^{2}\right) .
\end{aligned}
$$

As a result, the conditional signal-to-interference-plusnoise ratio (SINR) of $R_{k}^{(q)}, \gamma_{\mathbf{u}_{k}}^{(q)}$, is given by $\gamma_{\mathbf{u}_{k}}^{(q)}=$ $\left|m_{R_{k}^{(q)}}\right|^{2} /\left(2 \sigma_{R_{k}^{(q)}}^{2}\right)$.

The MSE of $u_{k}^{(q)}$ is expressed as

$$
\sigma_{u_{k}^{(q)}}^{2}=\int_{-\pi}^{\pi}\left(u_{k}^{(q)}\right)^{2} f_{u_{k}^{(q)}}\left(u_{k}^{(q)}\right) \mathrm{d} u_{k}^{(q)},
$$

where $f_{u_{k}^{(q)}}\left(u_{k}^{(q)}\right)$ is the unconditional pdf of $u_{k}^{(q)}$. Exact evaluation or deriving even the modified Cramer-Rao bound [28] for $\sigma_{u_{k}^{(q)}}^{2}$ is infeasible since $f_{u_{k}^{(q)}}\left(u_{k}^{(q)}\right)$ is unavailable [20].

To obtain a deep insight into the MSE, we present a recursive MSE lower bound, $B\left(\sigma_{u_{k}^{(q)}}^{2}\right)$, as follows (see Appendix).

$$
B\left(\sigma_{u_{k}^{(q)}}^{2}\right)=\frac{1}{2 \bar{\gamma}_{k}^{(q)}}-\frac{1}{\operatorname{erf}\left(\sqrt{\bar{\gamma}_{k}^{(q)}} \pi\right)} \sqrt{\frac{\pi}{\bar{\gamma}_{k}^{(q)}}} \exp \left(-\bar{\gamma}_{k}^{(q)} \pi^{2}\right),
$$

where $\bar{\gamma}_{k}^{(q)}=\mathbb{E}_{\mathbf{u}_{k}}\left(\gamma_{\mathbf{u}_{k}}^{(q)}\right)$ is the average SINR of $R_{k}^{(q)}$, and can be recursively calculated by

$$
\bar{\gamma}_{k}^{(q)}= \begin{cases}\frac{J p K\left|P_{u}\left(\phi_{k}\right)\right|^{2}}{2\left[J p+(1+K) \sigma_{n}^{2}\right]} & q=1 \\ \bar{\gamma}_{k}^{(q-1)}+\frac{\bar{\gamma}_{k}^{(1)} \sqrt{\bar{\gamma}_{k}^{(q-1)} / \pi}}{N \operatorname{erf}\left(\sqrt{\bar{\gamma}_{k}^{(q-1)}} \pi\right)} \int_{-\pi}^{\pi} \frac{\sin ^{2}\left(N u_{k}^{(q-1)}\right)}{\sin ^{2}\left(u_{k}^{(q-1)}\right)} & \\ \cdot \exp \left(-\bar{\gamma}_{k}^{(q-1)}\left(u_{k}^{(q-1)}\right)^{2}\right) d u_{k}^{(q-1)}, & q>1\end{cases}
$$

\section{Simulation Results}

In this section, we present the simulation results for the proposed schemes and channel estimation method using an interleaved hybrid array with $M=4$ and $N=16$. We assume that each user equipped with eight antennas can estimate its AoA in LOS directions and configure the corresponding BF vector perfectly in Step 1. Each user's signal experiences multipath fading, where the number of multipath is 8 with one dominating LOS-path and 7 NLOS-paths [12], [29]. The AoAs of all users' signals at both the BS and users sides are uniformly distributed in the range $[-\pi / 2, \pi / 2]$. Unless otherwise specified, the default values used in simulations are as follows: The number of users $J=4$, the Rician factor $K=15 \mathrm{~dB} \forall k=0, \ldots, J-1$, the updating coefficient $\mu=0.001$ and $p / \sigma_{n}^{2}=-5 \mathrm{~dB}$.

\section{A. Achievable Sum-Rate Comparison Between Proposed and SIC Schemes}

With perfect CSI, we compare the achievable sum-rates of the proposed schemes I-III, with the benchmarking schemes (a) and (b). Fig. 4 shows the achievable sum-rate versus $\frac{p}{\sigma_{n}^{2}}$ under $K=2 \mathrm{~dB}$. It is observed from Fig. 4 that the proposed scheme III can achieve about $93 \%$ and even $98 \%$ of the sum-rates achieved by the SIC schemes $(a)$ and $(b)$ under $\frac{p}{\sigma_{n}^{2}}=-5 \mathrm{~dB}$, respectively. The proposed scheme III achieves better performance than the schemes I and II. For comparison, the random subarray allocation is studied, where the subarrays are randomly assigned to different users. As shown in Fig. 4, the proposed ones outperform the random subarray allocation in terms of achievable sum-rate through allocation optimization. In addition, the localized array leads to slightly better performance than the interleaved one. 


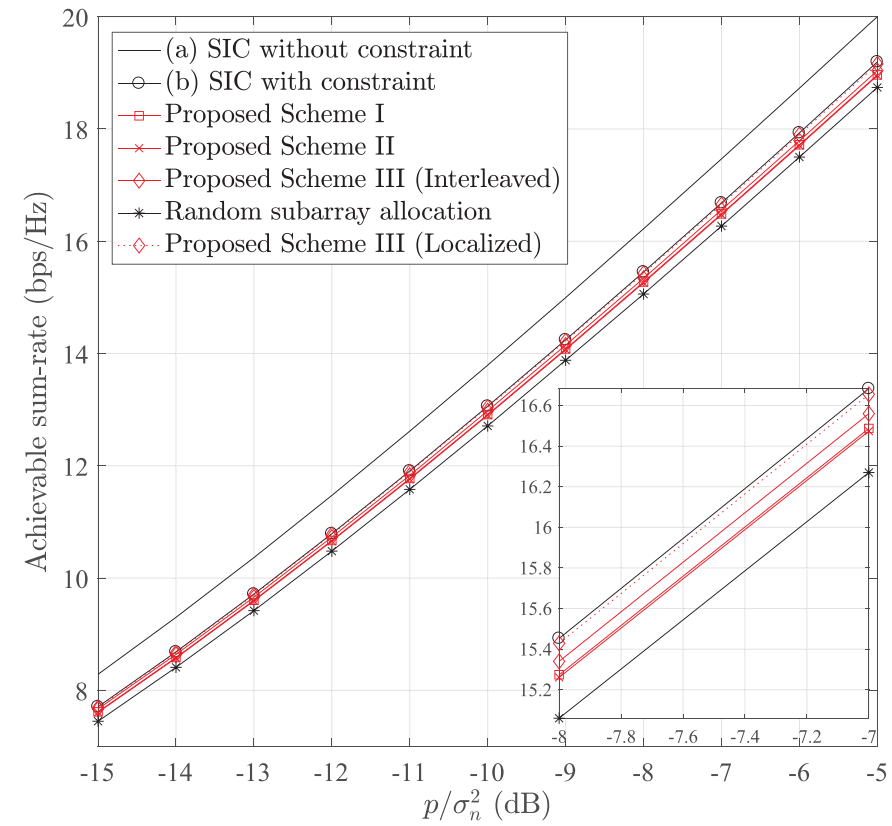

Fig. 4. Achievable sum-rate (bps/Hz) versus $\frac{p}{\sigma_{n}^{2}}(\mathrm{~dB})$.

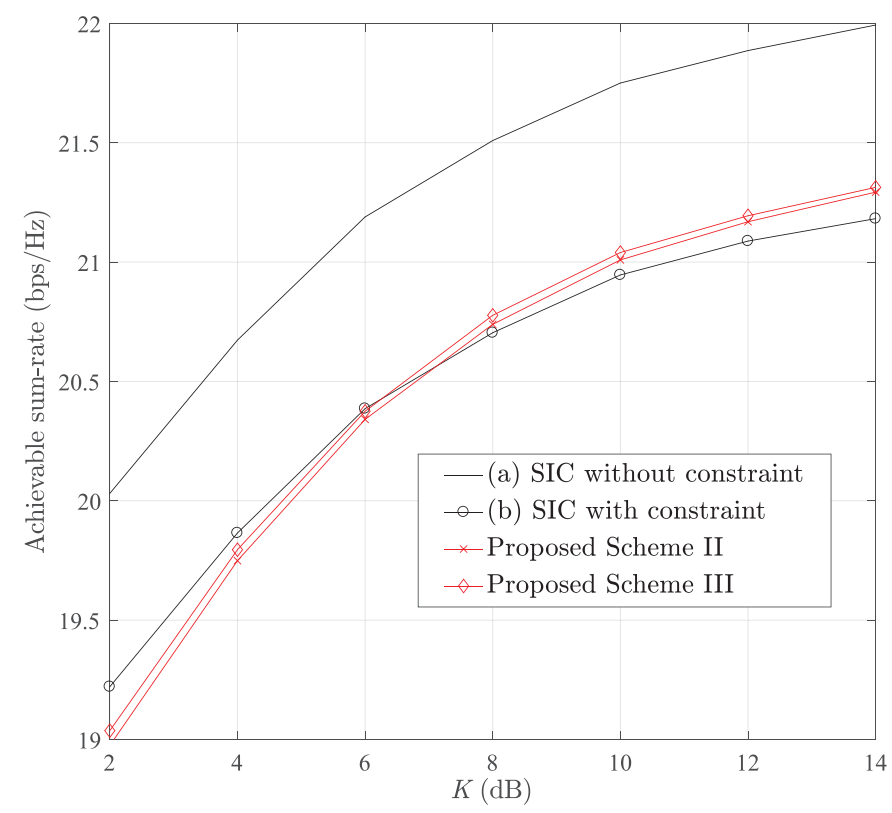

Fig. 5. Achievable sum-rate $(\mathrm{bps} / \mathrm{Hz})$ versus $K(\mathrm{~dB})$.

Fig. 5 shows the achievable sum-rates against the Rician factor $K$, where only the results for Scheme II are presented due to the similar performance between Schemes I and II. It is seen from Fig. 5 that with increasing $K$, the achievable sum-rates increase for all curves, while the gap between the proposed ones and the SIC scheme $(a)$ becomes smaller. The scheme III achieves higher sum-rate than scheme II, which is in line with the results in Fig. 4. Particularly, the proposed schemes even outperform the SIC scheme $(b)$ when the Rician factor, $K$, is larger than $7 \mathrm{~dB}$. When $K$ is small, the sum-rates of the proposed schemes are reduced since the signal power in the dominating LOS direction is reduced and the users suffer from more mutual interference from NLOS paths. Compara-

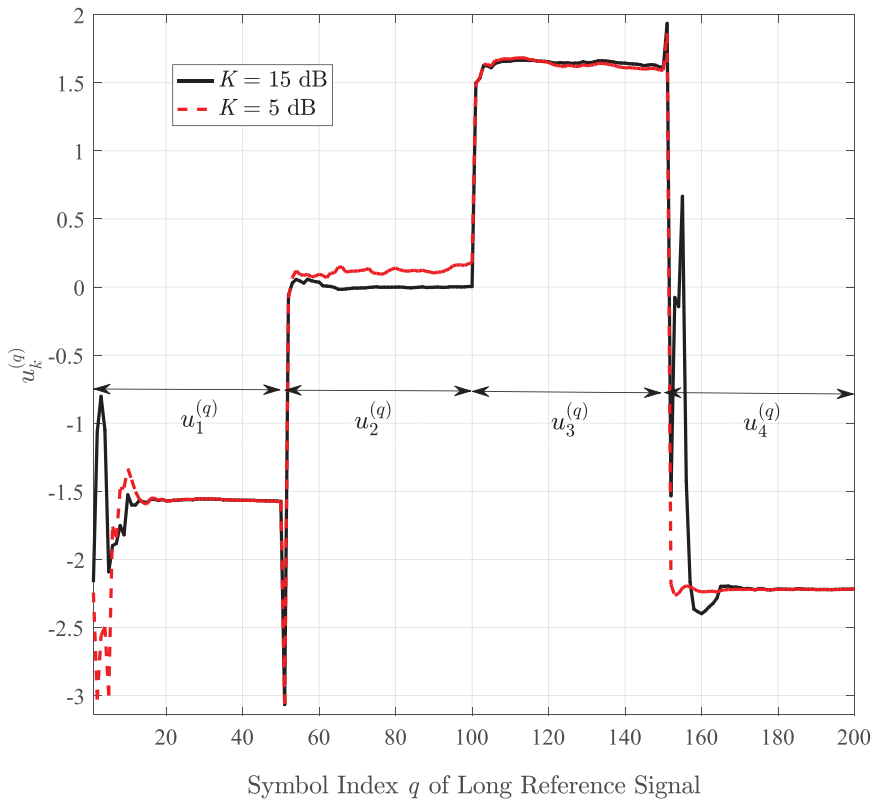

Fig. 6. $u_{k}^{(q)}$ versus the symbol index of long reference signal.

tively, the sum-rates of schemes $(a)$ and $(b)$ are less affected due to the SIC processing. This indicates that our proposed scheme is more suitable for LOS-dominated channels.

\section{B. Performance of the Proposed Channel Estimation Method}

Fig. 6 shows the performance of multiuser AoA estimation versus the symbol index of long reference signal under $\frac{p}{\sigma_{n}^{2}}=$ $-10 \mathrm{~dB}$, where four users' AoAs are assumed to be $\theta_{0}^{n}=$ $-\pi / 6, \theta_{1}=0, \theta_{2}=\pi / 6$ and $\theta_{3}=-\pi / 4$ (corresponding to $u_{0}=-1.5708, u_{1}=0, u_{2}=1.5708$ and $\left.u_{3}=-2.2214\right)$, respectively. It is observed from Fig. 6 that each user's AoA information can be iteratively estimated using its allocated nonzero symbols $\left(\frac{Q}{J}=50\right)$ in the reference signal $(Q=200)$. The AoA estimates under $K=15 \mathrm{~dB}$ have better convergence and accuracy performance than those under $K=5 \mathrm{~dB}$, because a higher Rician factor leads to a higher SINR. The corresponding estimates for all users' AoAs after 50 nonzero symbols are given by $-1.574,0.0035,1.6152$ and -2.2173 , respectively.

We evaluate the MSEs of the AoA estimates for a randomly selected user with different values of $K$ as the length of nonzero long reference signal increases. The results are obtained by averaging over 5000 independent simulations. As shown in Fig. 7, the MSEs decrease with the number of nonzero symbols. When the number of nonzero symbols is 50 , an MSE of 0.00456 can be achieved with $K=15 \mathrm{~dB}$, and it is about half of that achievable by using 30 nonzero symbols. The calculated recursive MSE bounds are also displayed in the same figure. We see that the MSE bounds become tighter as $K$ increases.

Fig. 8 shows the calculated and simulated MSEs for the equivalent channel estimation in Step 3 versus $p / \sigma_{n}^{2}$ under different lengths of short reference signals. As shown in the figure, the simulated MSEs match the calculated results in (11), and linearly decrease with the increase of $p / \sigma_{n}^{2}$ for the 


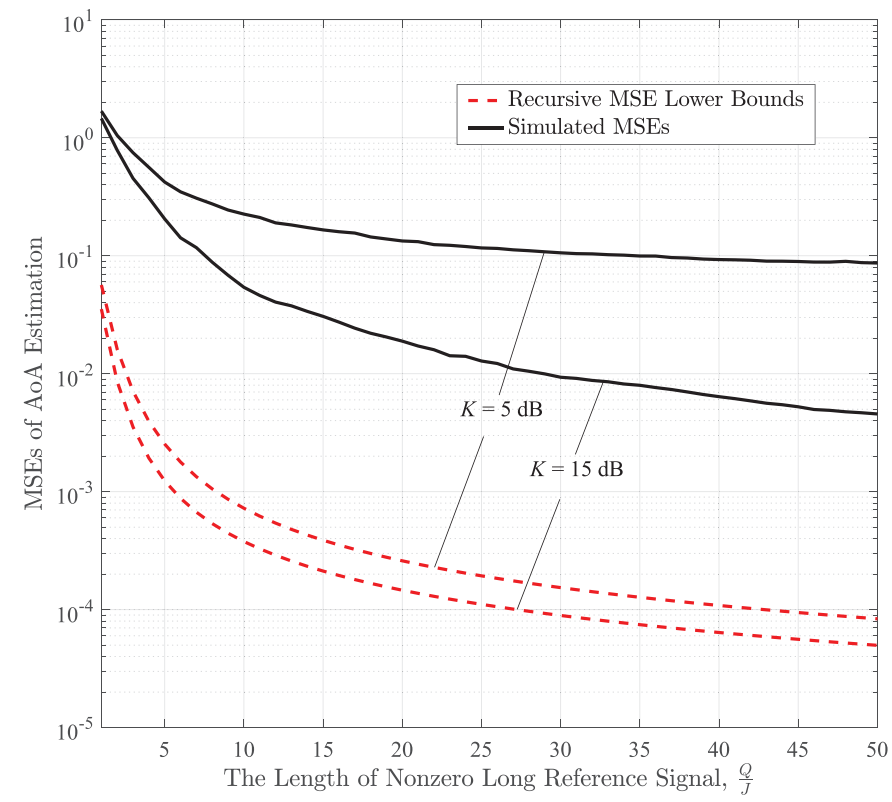

Fig. 7. Simulated MSEs and the recursive MSE lower bounds under $K=5$ and $15 \mathrm{~dB}$.

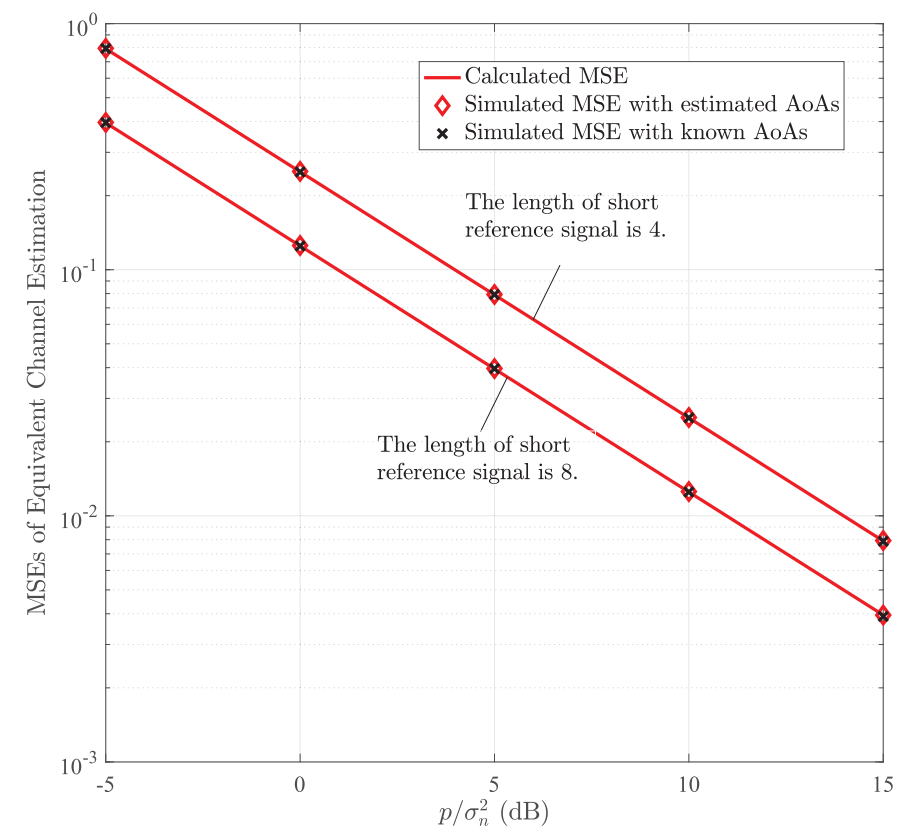

Fig. 8. Calculated and simulated MSEs for equivalent channel estimation.

cases with both estimated and known AoAs. It is also shown that the MSE performance can be improved by increasing the length of short reference signals.

Finally, we demonstrate the achievable sum-rate after ZF equalization using the proposed channel estimation method, in which the nonzero symbol length of each user's long reference signal is set to 50. Fig. 9 presents its performance as a function of $p / \sigma_{n}^{2}$ ranging from -5 to $5 \mathrm{~dB}$. As a comparison, the achievable sum-rates under perfect AoA and equivalent channel estimations are also plotted. It is shown that, the curves of sum-rate for the proposed channel estimation method are getting closer to those for perfectly known channels with increasing $K$. When $K$ is increased to $15 \mathrm{~dB}$, the proposed channel estimation method only incurs a

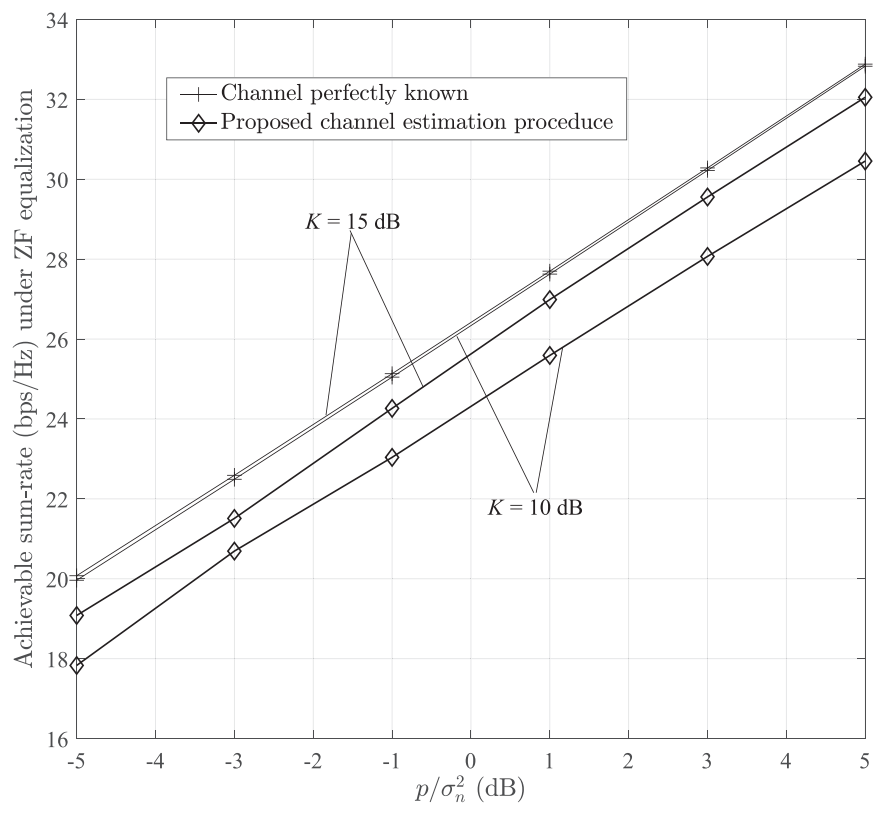

Fig. 9. Achievable sum-rate under $\mathrm{ZF}$ equalization under $K=10$ and $15 \mathrm{~dB}$.

$0.5 \mathrm{~dB}$ loss compared to perfectly known channels, achieving a sum rate of $30 \mathrm{bps} / \mathrm{Hz}$. While $K$ is from $10 \mathrm{~dB}$ to $15 \mathrm{~dB}$, there will be a $5.24 \%$ sum-rate gain at $p / \sigma_{n}^{2}=5 \mathrm{~dB}$. This indicates that the proposed channel estimation method is suitable for a LOS dominated mmWave channel.

\section{CONCLUSion}

We have proposed a user-directed multiuser receiver with three allocation schemes of subarrays to users for massive hybrid array mmWave communications. The receiver only needs users' AOA information from the dominating LOS paths and a small amount of equivalent channel information instead of the full CSI. This leads to a low-complexity implementation of analog BF and channel estimation, and also avoids excessive searching and feedback overhead. We have designed long reference signals to enable the receiver to estimate all users' dominating AOAs, which are used to generate the analog BF. The recursive MSE lower bounds for the AoA estimation in typical mmWave channels are derived. The simulated MSEs show that the performance of AoA estimation can be improved by increasing the nonzero symbol length of long reference signals. An equivalent channel estimation approach along with the designed short reference signals is then proposed. Simulation results demonstrate that the proposed schemes along with the channel estimation method can effectively trade off the sum-rate against the implementation complexity, which is particularly promising for massive hybrid arrays in LOS dominated channels.

The potential of the proposed user-directed multi-user receiver for future wireless networks lies in its feasibility and scalability in massive arrays due to the partially-connected array structure. Compared with the fully-connected structure, it can greatly reduce the number of phase shifters and thus hardware implementation complexity in practical deployment. Our proposed schemes in this paper focus on the optimization of allocating subarrays to users by iteratively finding the 
maximal equivalent channel gain between the subarrays and users. Other effective allocation approaches based on different optimization criteria may be investigated to further improve the performance of the schemes. In addition, some robust AoA estimation approaches, e.g., [30], [32] can be potentially used to improve the accuracy of AoA estimation in the proposed channel estimation method.

\section{APPENDIX}

DERIVATION OF (22)

The term $\frac{1}{L} \sum_{l=1}^{L}\left|P_{s}\left(\Delta u_{k, l}^{(i-1)}\right) P_{u}\left(\phi_{k, l}\right)\right|^{2}$ in $\sigma_{R_{k}^{(q)}}^{2}$ can be approximated by taking the expectation with respect to (w.r.t.) $u_{k, l}$ and $\phi_{k, l}$ as

$$
\begin{aligned}
& \frac{1}{L} \sum_{l=1}^{L}\left|P_{s}\left(\Delta u_{k, l}^{(i-1)}\right) P_{u}\left(\phi_{k, l}\right)\right|^{2} \\
& \approx \mathbb{E}_{u_{k, l}, \phi_{k, l}}\left\{\left|P_{s}\left(\Delta u_{k, l}^{(i-1)}\right) P_{u}\left(\phi_{k, l}\right)\right|^{2}\right\} \\
& \stackrel{(a)}{=} \mathbb{E}_{u_{k, l}}\left\{\left|P_{s}\left(\Delta u_{k, l}^{(i-1)}\right)\right|^{2}\right\} \cdot \mathbb{E}_{\phi_{k, l}}\left\{\left|P_{u}\left(\phi_{k, l}\right)\right|^{2}\right\} \stackrel{(b)}{=} 1,
\end{aligned}
$$

where (a) holds assuming $u_{k, l}$ and $\phi_{k, l}$ are independent, and (b) holds since $\mathbb{E}_{u_{k, l}}\left\{\left|P_{s}\left(\Delta u_{k, l}^{(i-1)}\right)\right|^{2}\right\}=$ $\mathbb{E}_{\phi_{k, l}}\left\{\left|P_{u}\left(\phi_{k, l}\right)\right|^{2}\right\}=1$ by exploiting the property of discrete-time Fourier transform in Appendix D [30]. By substituting (24) into $\sigma_{R_{k}^{(q)}}^{2}$ and taking the expectation of $\gamma_{\mathbf{u}_{k}}^{(q)}$ w.r.t. $\mathbf{u}_{k}$, we get the average SINR of $R_{k}^{(q)}$ as

$$
\bar{\gamma}_{k}^{(q)}=\frac{J p K\left|P_{u}\left(\phi_{k}\right)\right|^{2}}{2\left[J p+(1+K) \sigma_{n}^{2}\right]} \sum_{i=1}^{q} \mathbb{E}_{u_{k}^{(i-1)}}\left\{\left|P_{s}\left(\Delta u_{k}^{(i-1)}\right)\right|^{2}\right\} .
$$

To obtain a lower MSE bound, we replace $f_{u_{k}^{(q)}}\left(u_{k}^{(q)}\right)$ with a known phase distribution determined by $\bar{\gamma}_{k}^{(q)}$, i.e., $f_{u_{k}^{(q)}}\left(u_{k}^{(q)}\right)=f_{0}\left(\Delta u_{k}^{(q)}, \bar{\gamma}_{k}^{(q)}\right)[31]$, where

$$
f_{0}(x, \gamma) \approx \frac{1}{\operatorname{erf}(\sqrt{\gamma} \pi)} \sqrt{\frac{\gamma}{\pi}} \exp \left(-\gamma x^{2}\right), \quad-\pi \leq x \leq \pi,
$$

and $\operatorname{erf}(x)=\frac{2}{\sqrt{\pi}} \int_{0}^{x} e^{-t^{2}} d t$ is the error function. When $P_{s}\left(\Delta u_{k}^{(q)}\right)$ takes the maximum value at $u_{k}^{(q)}=u_{k}$, $f_{0}\left(\Delta u_{k}^{(q)}, \bar{\gamma}_{k}^{(q)}\right)$ can be considered as the true phase pdf. However, $\left|P_{s}\left(\Delta u_{k}^{(q)}\right)\right| \leq \sqrt{N}$ when there exists recursive nuisance parameter, which somewhat reduces the SINR. Hence, the actual MSE in (21) will always be larger than that calculated using $f_{0}\left(\Delta u_{k}^{(q)}, \bar{\gamma}_{k}^{(q)}\right)$, i.e.,

$$
\sigma_{u_{k}^{(q)}}^{2} \geq \int_{-\pi}^{\pi}\left(u_{k}^{(q)}\right)^{2} f_{0}\left(u_{k}^{(q)}, \bar{\gamma}_{k}^{(q)}\right) d u_{k}^{(q)}=B\left(\sigma_{u_{k}^{(q)}}^{2}\right) .
$$

From $(25), \bar{\gamma}_{k}^{(1)}$ can be determined as

$$
\begin{aligned}
\bar{\gamma}_{k}^{(1)} & =\frac{J p K\left|P_{u}\left(\phi_{k}\right)\right|^{2}}{2\left[J p+(1+K) \sigma_{n}^{2}\right]} \mathbb{E}_{u_{k}^{(0)}}\left\{\left|P_{s}\left(\Delta u_{k}^{(0)}\right)\right|^{2}\right\} \\
& =\frac{J p K\left|P_{u}\left(\phi_{k}\right)\right|^{2}}{2\left[J p+(1+K) \sigma_{n}^{2}\right]},
\end{aligned}
$$

where the initial estimate $u_{k}^{(0)}$ is assumed to be uniformly distributed in $[-\pi, \pi)$, and thus $\bar{\gamma}_{k}^{(q)}$ for $q>1$ can be recursively determined as

$$
\begin{aligned}
\bar{\gamma}_{k}^{(q)}= & \bar{\gamma}_{k}^{(q-1)}+\frac{J p K\left|P_{u}\left(\phi_{k}\right)\right|^{2}}{2\left[J p+(1+K) \sigma_{n}^{2}\right]} \\
& \cdot \int_{-\pi}^{\pi}\left|P_{s}\left(\Delta u_{k}^{(q-1)}\right)\right|^{2} f_{0}\left(u_{k}^{(q-1)}, \bar{\gamma}_{k}^{(q-1)}\right) d u_{k}^{(q-1)}
\end{aligned}
$$

By substituting (26) into (27) and (29), (22) can be obtained.

\section{REFERENCES}

[1] R. W. Heath, N. González-Prelcic, S. Rangan, W. Roh, and A. M. Sayeed, "An overview of signal processing techniques for millimeter wave MIMO systems," IEEE J. Sel. Topics Signal Process., vol. 10 , no. 3, pp. 436-453, Apr. 2016.

[2] J. A. Zhang, X. Huang, V. Dyadyuk, and Y. J. Guo, "Massive hybrid antenna array for millimeter-wave cellular communications," IEEE Wireless Commun., vol. 22, no. 1, pp. 79-87, Feb. 2015.

[3] W. Roh et al., "Millimeter-wave beamforming as an enabling technology for $5 \mathrm{G}$ cellular communications: Theoretical feasibility and prototype results," IEEE Commun. Mag., vol. 52, no. 2, pp. 106-113, Feb. 2014.

[4] T. S. Rappaport et al., "Millimeter wave mobile communications for 5G cellular: It will work!" IEEE Access, vol. 1, pp. 335-349, May 2013.

[5] F. Sohrabi and W. Yu, "Hybrid digital and analog beamforming design for large-scale antenna arrays," IEEE J. Sel. Topics Signal Process. vol. 10 , no. 3, pp. 501-513, Apr. 2016.

[6] A. Alkhateeb, J. Mo, N. Gonzalez-Prelcic, and R. W. Heath, Jr., "MIMO precoding and combining solutions for millimeter-wave systems," IEEE Commun. Mag., vol. 52, no. 12, pp. 122-131, Dec. 2014.

[7] W. Ni and X. Dong, "Hybrid block diagonalization for massive multiuser MIMO systems," IEEE Trans. Commun., vol. 64, no. 1, pp. 201-211, Jan. 2016.

[8] O. El Ayach, S. Rajagopal, S. Abu-Surra, Z. Pi, and R. W. Heath, Jr., "Spatially sparse precoding in millimeter wave MIMO systems," IEEE Trans. Wireless Commun., vol. 13, no. 3, pp. 1499-1513, Mar. 2014.

[9] D. Ying, F. W. Vook, T. A. Thomas, and D. J. Love, "Hybrid structure in massive MIMO: Achieving large sum rate with fewer RF chains," in Proc. IEEE Intern. Commun. Conf. (ICC), Jun. 2015, pp. 2344-2349.

[10] L. Liang, W. Xu, and X. Dong, "Low-complexity hybrid precoding in massive multiuser MIMO systems," IEEE Wireless Commun. Lett., vol. 3, no. 6, pp. 653-656, Dec. 2014.

[11] A. Alkhateeb, G. Leus, and R. W. Heath, "Limited feedback hybrid precoding for multi-user millimeter wave systems," IEEE Trans. Wireless Commun., vol. 14, no. 11, pp. 6481-6494, Nov. 2015.

[12] L. Zhao, D. W. K. Ng, and J. Yuan, "Multi-user precoding and channel estimation for hybrid millimeter wave systems," IEEE J. Sel. Areas Commun., vol. 35, no. 7, pp. 1576-1590, Jul. 2017.

[13] A. Alkhateeb, O. El Ayach, G. Leus, and R. W. Heath, Jr., "Channel estimation and hybrid precoding for millimeter wave cellular systems," IEEE J. Sel. Topics Signal Process., vol. 8, no. 5, pp. 831-846, Oct. 2014.

[14] X. Gao, L. Dai, S. Han, C.-L. I, and X. Wang, "Reliable beamspace channel estimation for millimeter-wave massive MIMO systems with lens antenna array," IEEE Trans. Wireless Commun., vol. 16, no. 9, pp. 6010-6021, Sep. 2017.

[15] L. Dai, X. Gao, J. Quan, S. Han, and C.-L. I, "Near-optimal hybrid analog and digital precoding for downlink mmWave massive MIMO systems," in Proc. IEEE Int. Conf. Commun. (ICC), Jun. 2015 pp. 1334-1339.

[16] X. Gao, L. Dai, S. Han, I. Chih-Lin, and R. W. Heath, "Energy-efficient hybrid analog and digital precoding for MmWave MIMO systems with large antenna arrays," IEEE J. Sel. Areas Commun., vol. 34, no. 4, pp. 998-1009, Apr. 2016

[17] J. A. Zhang, H. Li, X. Huang, Y. Guo, and A. Cantoni, "Userdirected analog beamforming for multiuser millimeter-wave hybrid array systems," in Proc. IEEE Veh. Technol. Conf. (VTC), Jun. 2017, pp. 1-5.

[18] Y. Zhu, Q. Zhang, and T. Yang, "Low-complexity hybrid precoding with dynamic beam assignment in mmWave OFDM systems," IEEE Trans. Veh. Technol., vol. 67, no. 4, pp. 3685-3689, Apr. 2018.

[19] H. Li, Q. Wang, X. Huang, and J. A. Zhang, "Low-complexity uplink multiuser receivers for MIMO system with massive hybrid array," in Proc. IEEE Veh. Technol. Conf. (VTC), Jun. 2017, pp. 1-5. 
[20] X. Huang, Y. J. Guo, and J. D. Bunton, "A hybrid adaptive antenna array," IEEE Trans. Wireless Commun., vol. 9, no. 5, pp. 1770-1779, May 2010.

[21] W. Rhee and J. M. Cioffi, "On the capacity of multiuser wireless channels with multiple antennas," IEEE Trans. Inf. Theory, vol. 49, no. 10 , pp. $2580-2595$, Oct. 2003.

[22] A. Goldsmith, S. A. Jafar, N. Jindal, and S. Vishwanath, "Capacity limits of MIMO channels," IEEE J. Sel. Areas Commun., vol. 21, no. 5, pp. 684-702, Jun. 2003.

[23] S. Buzzi and C. D'Andrea, "Doubly massive mmWave MIMO systems: Using very large antenna arrays at both transmitter and receiver," in Proc. IEEE Global Commun. Conf. (GLOBECOM), Dec. 2016, pp. 1-6.

[24] A. Alkhateeb and R. W. Heath, Jr., "Frequency selective hybrid precoding for limited feedback millimeter wave systems," IEEE Trans. Commun., vol. 64, no. 5, pp. 1801-1818, May 2016.

[25] X. Huang and Y. J. Guo, "Frequency-domain AoA estimation and beamforming with wideband hybrid arrays," IEEE Trans. Wireless Commun., vol. 10, no. 8, pp. 2543-2553, Aug. 2011.

[26] S. Hur, T. Kim, D. J. Love, J. V. Krogmeier, T. A. Thomas, and A. Ghosh, "Millimeter wave beamforming for wireless backhaul and access in small cell networks," IEEE Trans. Commun., vol. 61, no. 10, pp. 4391-4403, Oct. 2013.

[27] A. Papoulis and S. U. Pillai, Probability, Random Variables and Stochastic Processes, 4th ed. New York, NY, USA: McGraw-Hill, 2002.

[28] A. N. D'Andrea, U. Mengali, and R. Reggiannini, "The modified Cramer-Rao bound and its application to synchronization problems," IEEE Trans. Commun., vol. 42, nos. 2-4, pp. 1391-1399, Feb./Apr. 1994.

[29] L. Zhao, Z. Wei, D. W. K. Ng, J. Yuan, and M. C. Reed, "Multi-cell hybrid millimeter wave systems: Pilot contamination and interference mitigation," IEEE Trans. Commun., vol. 66, no. 11, pp. 5740-5755, Nov. 2018.

[30] K. Wu, W. Ni, T. Su, R. P. Liu, and Y. J. Guo, "Robust unambiguous estimation of angle-of-arrival in hybrid array with localized analog subarrays," IEEE Trans. Wireless Commun., vol. 17, no. 5, pp. 2987-3002, May 2018.

[31] X. Huang and Y. J. Guo, "MSE lower bounds for phase estimation based on overlapped Gaussian distribution," in Proc. 10th Int. Symp. Commun. Inf. Technol. (ISCIT), Tokyo, Japan, Oct. 2010, pp. 26-29.

[32] J. A. Zhang, W. Ni, P. Cheng, and Y. Lu, "Angle-of-arrival estimation using different phase shifts across subarrays in localized hybrid arrays," IEEE Commun. Lett., vol. 20, no. 11, pp. 2205-2208, Nov. 2016.

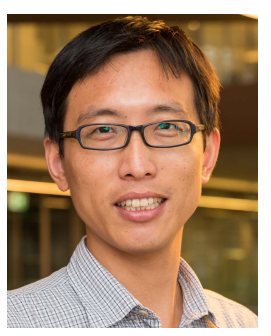

Hang Li received the B.Eng. and M.Eng. degrees from Beijing Jiaotong University, Beijing, China, in 2003 and 2006, respectively, and the Ph.D. degree from the University of Western Australia, Perth, WA, Australia, in 2014, all in electronic engineering. From 2016 to 2019, he was a Post-Doctoral Research Fellow with the Global Big Data Technologies Centre, University of Technology Sydney. His research interests include wireless and optical communications signal processing, wireless cross-layer protocols design, and mmWave massive MIMO.

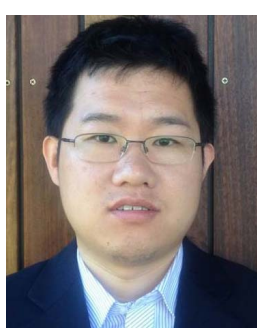

Thomas Q. Wang received the B.E. degree in electric engineering from Dalian Jiaotong University, Dalian, China, in 2006, and the M.E. and Ph.D. degrees in communication and information systems from Dalian Maritime University, Dalian, in 2008 and 2011, respectively. From 2012 to 2015, he was a Research Fellow with the Department of Electrical and Computer Systems Engineering, Monash University, Melbourne, VIC, Australia. From 2016 to 2019, he was a Research Fellow with the Global Big Data Technologies Centre, University of Technology Sydney, Sydney, NSW, Australia. His research interests include optical wireless communications and multiple-input multiple-output technology.

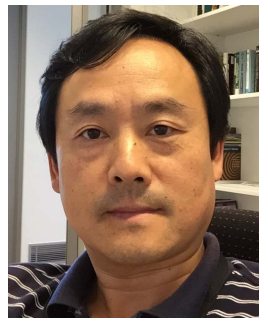

Xiaojing Huang (M'99-SM'11) received the B.Eng., M.Eng., and Ph.D. degrees in electronic engineering from Shanghai Jiao Tong University, Shanghai, China, in 1983, 1986, and 1989, respectively. He was a Principal Research Engineer with the Motorola Australian Research Center, Botany, NSW, Australia, from 1998 to 2003, and an Associate Professor with the University of Wollongong, Wollongong, NSW, Australia, from 2004 to 2008. He has been a Principal Research Scientist with the Commonwealth Scientific and Industrial Research Organisation (CSIRO), Sydney, NSW, Australia, and the Project Leader of the CSIRO Microwave and mm-Wave Backhaul projects since 2009. He is currently a Professor of information and communications technology with the School of Electrical and Data Engineering and the Program Leader for Mobile Sensing and Communications with the Global Big Data Technologies Center, University of Technology Sydney, Sydney, NSW, Australia. With over 30 years of combined industrial, academic, and scientific research experience, he has authored over 300 book chapters, refereed journal and conference papers, major commercial research reports, and filed 31 patents. He was a recipient of the CSIRO Chairman's Medal and the Australian Engineering Innovation Award in 2012 for exceptional research achievements in multigigabit wireless communications.

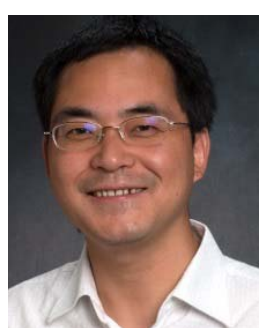

J. Andrew Zhang (M'04-SM'11) received the B.Sc. degree from Xi' an JiaoTong University, China, in 1996, the M.Sc. degree from the Nanjing University of Posts and Telecommunications, China, in 1999, and the Ph.D. degree from Australian National University in 2004.

$\mathrm{He}$ was a Researcher with Data61, CSIRO, Australia, from 2010 to 2016, the Networked Systems, NICTA, Australia, from 2004 to 2010, and ZTE Corp., Nanjing, China, from 1999 to 2001. $\mathrm{He}$ is currently an Associate Professor with the School of Electrical and Data Engineering, University of Technology Sydney, Australia. He has published more than 130 papers in leading international journals and conference proceedings. His research interests include the area of signal processing for wireless communications and sensing and autonomous vehicular networks. He has received four best paper awards for his work. He was a recipient of CSIRO Chairman's Medal and the Australian Engineering Innovation Award in 2012 for exceptional research achievements in multi-gigabit wireless communications.

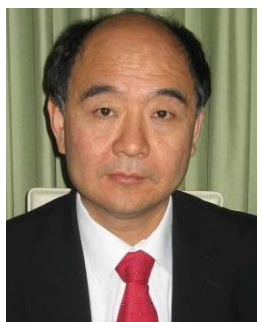

Y. Jay Guo (F'14) received the bachelor's and master's degrees from Xidian University, China, in 1982 and 1984, respectively, and the Ph.D. degree from Xian Jiaotong University, China, in 1987. He has published over 400 research papers and holds 24 patents in antennas and wireless systems. His research interests include antennas, mm-wave and $\mathrm{THz}$ communications and sensing systems and big data technologies. He is a fellow of the Australian Academy of Engineering and Technology, IEEE, and IET. He is a member of the College of Experts of the Australian Research Council. He has received a number of most prestigious Australian national awards, and was named one of the most influential engineers in Australia in 2014 and 2015.

$\mathrm{He}$ is currently a Distinguished Professor and the Founding Director of the Global Big Data Technologies Centre, University of Technology Sydney, Australia. Prior to this appointment in 2014, he served as the Director with CSIRO for over nine years, directing a number of ICT research portfolios. Before joining CSIRO, he held various senior technology leadership positions in Fujitsu, Siemens, and NEC, U.K.

Prof. Guo has chaired numerous international conferences. He is the Chair-Elect of the International Steering Committee and the International Symposium on Antennas and Propagation (ISAP). He was the International Advisory Committee Chair of the IEEE VTC2017, the General Chair of ISAP2015, iWAT2014 and WPMC'2014, and the TPC Chair of 2010 IEEE WCNC, and 2012 and 2007 IEEE ISCIT. He served as the Guest Editor of Special Issues on Antennas for Satellite Communications and Antennas and Propagation Aspects of 60-90GHz. Wireless Communications, both in the IEEE Trans actions on ANTENNAS AND Propagation, Special Issue on Communications Challenges and Dynamics for Unmanned Autonomous Vehicles, the IEEE JOURnAL ON SELECTED AREAS IN COMMUNICATIONS, and Special Issue on $5 G$ for Mission Critical Machine Communications, IEEE Network Magazine. 